\title{
SKEIN THEORY AND TOPOLOGICAL QUANTUM REGISTERS: BRAIDING MATRICES AND TOPOLOGICAL ENTANGLEMENT ENTROPY OF NON-ABELIAN QUANTUM HALL STATES
}

\author{
KAZUHIRO HIKAMI
}

\begin{abstract}
We study topological properties of quasi-particle states in the non-Abelian quantum Hall states. We apply a skein-theoretic method to the Read-Rezayi state whose effective theory is the $S U(2)_{K}$ Chern-Simons theory. As a generalization of the Pfaffian $(K=2)$ and the Fibonacci $(K=3)$ anyon states, we compute the braiding matrices of quasi-particle states with arbitrary spins. Furthermore we propose a method to compute the entanglement entropy skein-theoretically. We find that the entanglement entropy has a nontrivial contribution called the topological entanglement entropy which depends on the quantum dimension of non-Abelian quasi-particle intertwining two subsystems.
\end{abstract}

\section{INTRODUCTION}

Since the discovery of the fractional Hall effect and the Laughlin wave function, it has been extensively studied the fractional statistics, i.e., anyon (see e.g. Ref. 59). The effective theory of the Laughlin wave function for $\nu=\frac{1}{2 m+1}$ is the $U(1)$ Chern-Simons (CS) theory [50, 64], and those quasi-particles as excited state are understood as the Abelian anyon. Contrary to these fractional quantum Hall states, the $\nu=5 / 2$ Hall state experimentally observed in Ref. 60 remains mysterious (see e.g. Ref. 51). One of theoretical candidates for this state is the Moore-Read Pfaffian state [44], which is related to the BCS ground state wave function of the $p+\mathrm{i} p$ superconductor such as $\mathrm{Sr}_{2} \mathrm{RuO}_{4}$ [24, 52]. The effective theory of the Pfaffian state was identified with the $S U(2)_{2}$ CS theory [12], and the non-Abelian property and the dimension of the Hilbert space of the many-quasi-particle state have been studied from this viewpoint [46].

As a generalization of the Pfaffian state as the non-Abelian quantum Hall state, proposed is the Read-Rezayi state [53]. This state with $K=3$ is expected to describe the $\nu=12 / 5$ quantum Hall states reported in Ref. 62, and also pointed out is a relationship with the rotating Bose-Einstein condensates [8]. Originally the Read-Rezayi state was constructed by use of the $\mathbb{Z}_{K}$ parafermion theory [63] which is closely related to the $S U(2)_{K}$ CS theory. Algebraic structure of the ReadRezayi state was analyzed by use of the quantum group $U_{q}(S U(2))$, and the non-Abelian property as the braiding matrices of quasi-particle states was studied [56].

Date: September 14, 2007.

Key words and phrases. skein theory, Chern-Simons theory, topological quantum field theory, quantum invariant, non-Abelian quantum Hall state, Read-Rezayi state, Pfaffian state . 
The CS field theory plays an important role in the quantum topological invariant of links and 3manifolds. Witten constructed the quantum invariant of 3-manifolds from the Chern-Simons partition function with gauge group $G$ based on conformal block [45], and he further identified quantum polynomial invariants such as the Jones polynomial [29] and the HOMFLY polynomial [17] as the expectation value of the Wilson loop of the CS theory [61]. His construction in terms of path integral is not mathematically rigorous, and the invariant of 3-manifold was later constructed combinatorially by Reshetikhin and Turaev [54], and it is called the Witten-Reshetikhin-Turaev (WRT) invariant.

Recently from the viewpoint of the quantum computation (see e.g. Refs. 37, 47), the nonAbelian quantum Hall states have received renewed interests. As a fault-tolerant quantum computing system, Kitaev proposed to construct quantum registers topologically [34, 36]. Though his toric code uses a spin system on 2-dimensional honeycomb lattice as a non-Abelian quasi-particle system (see also Ref. 40 where topological phase of trivalent graph, sometimes called string net or spin network, is discussed), the quantum Hall system is another candidate for non-Abelian statistics (see Refs. 3, 9, 20 for recent reviews). In such topological quantum registers, unitary operations are constructed from braiding of non-Abelian anyons. It was shown [13, 14] that the braiding in the $S U(2)_{K}$ Chern-Simons theory can efficiently approximate, i.e., quantum compile, any quantum computing gate; $n_{\geq 3}$-braid group for $K \geq 3, K \neq 4,8$ and $n_{\geq 5}$-braid group for $K=8$ are capable of universal quantum computation.

One of our purposes in this article is to study the braiding property of non-Abelian quasiparticles in the $S U(2)_{K}$ CS theory. By use of explicit form of correlation function in Ref. 38, Ardonne and Schoutens studied braiding matrices [2] for 4-quasi-particle states. It becomes difficult to derive braiding matrices for many-quasi-particle states by their method. We rather use the Temperley-Lieb algebra or the skein theory, which is elementary and combinatorial, to derive the braiding matrices. Although, a naïve application of the skein theory does not give a unitary representation. We show explicitly how to construct the unitary braiding matrices by use of skein theory. See also Ref. 32, where proposed is a different method to construct the unitary operators from the skein theory by modifying a weight of trivalent vertex. Our method is essentially equivalent to that of Ref. 56, once we know both a construction of the colored Jones polynomial based on representation theory of the quantum group and a quantum group structure of the conformal field theory (see e.g. Refs. 23, 30). So most of methods in this article might be well known, except our new result is a computation of the entanglement entropy (see Refs. 1 for recent review) in the framework of the skein theory. In a topological phase such as non-Abelian states, it was discussed [35, 39] that the entanglement entropy has a topological part, which is called the topological entanglement entropy, and that it is expected to detect a topological order [58]. Some numerical and analytical studies have been done for the quantum dimer model in a triangular lattice [18], for the fractional quantum Hall states of the fermionic Laughlin type [25], and for the quantum eight-vertex model [48]. Here we show that the entanglement entropy of the state 
$|\Psi\rangle=\sum_{j} p_{j}\left|\psi_{j}\right\rangle_{A} \otimes\left|\phi_{j}\right\rangle_{B}$ with $\sum_{j}\left|p_{j}\right|^{2}=1$ is given by

$$
S_{A}=\sum_{j}\left|p_{j}\right|^{2} \log \left(\frac{d_{j}}{\left|p_{j}\right|^{2}}\right)
$$

which includes the topological entanglement entropy

$$
S_{A}^{\text {topo }}=\sum_{j}\left|p_{j}\right|^{2} \log d_{j}
$$

Here $d_{j}$ denotes the quantum dimension of quasi-particle which connects subspaces $A$ and $B$.

A content of this article is constructed as follows. In Section 2, we review the Read-Rezayi state. We shall discuss a relationship with the $\mathbb{Z}_{K}$ parafermion CFT, the $S U(2)_{K}$ Chern-Simons theory, and the $S U(2)$ topological quantum invariants. We further explain basic facts about the Temperley-Lieb skein theory following Ref. 31. In Section 3 we outline a method to compute the entanglement entropy of quasi-particle states skein-theoretically. We define the topological entanglement entropy from the von Neumann entropy. In Section 4 we construct a unitary representation of braid operators on spin-1/2 state in the $S U(2)_{K}$ CS theory. The $S U(2)_{2}$ theory corresponds to the Moore-Read Pfaffian state. We shall further compute the entanglement entropy of these states, and we show that it has a topological entropy which depends on the quantum dimension of quasi-particle. Section 5 is devoted to studies of spin-1 quasi-particle states in the $S U(2)_{K}$ theory. A case of $K=3$ is known as the Fibonacci anyon model. We construct the bases of quasi-particle states, and give explicit forms of the braiding matrices. We also calculate the topological entanglement entropy. In Section 6 we study a correlation function which is composed from different species of quasi-particles as another candidate of topological quantum registers. We give an elementary treatment of skein theory up to Section 6 as best as we can. Later in Section 7 we shall give a general setup for many-quasi-particle states in terms of the quantum $6 j$ symbol. The braiding matrices and the topological entanglement entropy are discussed by use of the quantum $6 j$ symbol. The last section is devoted to concluding remarks and discussions.

\section{Non-Abelian Quantum Hall State, CFT, and Quantum Invariants}

\subsection{Parafermion Theory and the Read-Rezayi State}

The Read-Rezayi state is constructed by use of the $\mathbb{Z}_{K}$ parafermion theory [21, 63] (see also e.g. Ref. 10). The $\mathbb{Z}_{K}$ parafermion theory is equivalent to $S U(2)_{K} / U(1)$ theory, and we use the primary fields $\Phi_{m}^{\ell}$ with $-\ell<m \leq \ell$ and $\ell \in\{0,1, \ldots, K\}$ whose conformal dimension is

$$
h_{m}^{\ell}=\frac{\ell(\ell+2)}{4(K+2)}-\frac{m^{2}}{4 K}
$$

Here $m$ denotes the $\mathrm{U}(1)$ charge defined modulo $2 K$, and parameter $\ell$ parametrizes a spin- $\ell / 2$. The primary fields $G_{m}^{(\ell)}$ with $\ell, m \in \mathbb{Z} / 2$ of the $S U(2)_{K}$ theory is

$$
G_{m}^{(\ell)}=\Phi_{2 m}^{2 \ell} \exp \left(\frac{\mathrm{i} m}{\sqrt{K}} \varphi\right)
$$


where $\varphi$ is the free massless Bose field. Further for the field $\Phi_{m}^{\ell}$ we have $\ell=m \bmod 2$ and $\Phi_{m}^{\ell}=\Phi_{m+2 K}^{\ell}=\Phi_{m-K}^{K-\ell}$. The fusion rule follows from the $S U(2)_{K}$ theory as

$$
\Phi_{m}^{\ell} \times \Phi_{m^{\prime}}^{\ell^{\prime}}=\sum_{\ell^{\prime \prime}=\left|\ell-\ell^{\prime}\right|}^{\min \left(\ell+\ell^{\prime}, 2 K-\ell-\ell^{\prime}\right)} \Phi_{m+m^{\prime}}^{\ell^{\prime \prime}}
$$

In terms of the primary fields $\Phi_{m}^{\ell}$, the vacuum sector $I$ is $\Phi_{0}^{0}=\Phi_{K}^{K}=I$, and the parafermions $\psi_{\ell}$, the spin fields $\sigma_{\ell}$, the dual spin fields $\mu_{\ell}$ and neutral fields $\varepsilon_{\ell}$ are respectively defined by

$$
\begin{gathered}
\psi_{\ell}=\Phi_{2 \ell}^{0}=\Phi_{2 \ell-K}^{K} \\
\sigma_{\ell}=\Phi_{\ell}^{\ell} \\
\mu_{\ell}=\Phi_{-\ell}^{\ell} \\
\varepsilon_{\ell}=\Phi_{0}^{2 \ell}
\end{gathered}
$$

and we mean $\psi_{\ell}^{\dagger}=\psi_{K-\ell}$, and $\sigma_{\ell}^{\dagger}=\sigma_{K-\ell}$. Note that $h_{\psi_{\ell}}=\frac{\ell(K-\ell)}{K}, h_{\sigma_{\ell}}=h_{\mu_{\ell}}=\frac{\ell(K-\ell)}{2 K(K+2)}$, and $h_{\varepsilon_{\ell}}=\frac{\ell(\ell+1)}{K+2}$.

It is noted that the field $\psi_{1}$ for $K=2$ becomes the Majorana fermion $\psi$ satisfying $\langle\psi(z) \psi(w)\rangle=(z-w)^{-1}$, and the Pfaffian state is identified with the correlation function $\left\langle\psi\left(z_{1}\right) \cdots \psi\left(z_{N}\right)\right\rangle$ [44]. Correspondingly the wave function of the $n$-quasi-particle states can be constructed from the correlation function $\left\langle\sigma_{1}\left(w_{1}\right) \cdots \sigma_{1}\left(w_{n}\right) \psi\left(z_{1}\right) \cdots \psi\left(z_{N}\right)\right\rangle$. This function was studied in detail [46], and the dimension of the wave function for $2 n$-quasi-particle states are identified to be $2^{n-1}$.

The Read-Rezayi state [53] is a generalization of the Pfaffian state, and the wave function is defined as correlation function of the primary fields in the $\mathbb{Z}_{K}$ parafermion theory;

$$
\begin{aligned}
\Psi\left(w_{1}, \ldots, w_{n} ; z_{1}, \ldots, z_{N}\right) & =\left\langle\sigma_{1}\left(w_{1}\right) \cdots \sigma_{1}\left(w_{n}\right) \psi_{1}\left(z_{1}\right) \cdots \psi_{1}\left(z_{N}\right)\right\rangle \\
\times & \prod_{i<j}\left(z_{i}-z_{j}\right)^{M+\frac{2}{K}} \prod_{i=1}^{N} \prod_{j=1}^{n}\left(z_{i}-w_{j}\right)^{\frac{1}{K}} \prod_{i<j}\left(w_{i}-w_{j}\right)^{\frac{1}{K(K M+2)}}
\end{aligned}
$$

Here $M$ is an odd or even integer depending on whether the particles are fermions or bosons, and the filling factor becomes

$$
\nu=\frac{K}{K M+2}
$$

The fractional power in the second line of (2.4) was added to cancel the singular power which comes from the operator product expansion of $\Phi_{m}^{\ell}$ so that the wave function is non-singular. We should note that the Read-Rezayi state (2.4) without quasi-particles $(n=0)$ is an exact ground state of the Hamiltonian with $(K+1)$-body interactions;

$$
\mathcal{H}=V \sum_{i_{1}<i_{2} \cdots<i_{K+1}} \delta^{2}\left(z_{i_{1}}-z_{i_{2}}\right) \delta^{2}\left(z_{i_{2}}-z_{i_{3}}\right) \cdots \delta^{2}\left(z_{i_{K}}-z_{i_{K+1}}\right)
$$


So to study the action of braiding of quasi-particles on the wave function (2.4), we need the braiding actions on the correlation functions of parafermion fields. By use of correspondence (2.1) with the $S U(2)_{K}$ theory, we see that the braiding matrices coincide with that of the $S U(2)_{K}$ theory.

\subsection{Quantum Invariant from the Chern-Simons Theory}

In Ref. 61, the physical interpretation of the quantum knot invariant such as the Jones polynomial [29] and the HOMFLY polynomial [17] was given. The Chern-Simons action with gauge group $G$ on 3-manifold $\mathcal{M}$ is defined by

$$
S_{\mathrm{CS}}=\frac{K}{4 \pi} \int_{\mathcal{M}} \operatorname{Tr}\left(A \wedge \mathrm{d} A+\frac{2}{3} A \wedge A \wedge A\right)
$$

where $K$ is a coupling constant $K \in \mathbb{Z}$, and $A$ is the gauge connection on $\mathcal{M}$. Witten introduced the topological invariant of $\mathcal{M}$ as the partition function

$$
Z(\mathcal{M})=\int \mathcal{D} A \mathrm{e}^{\mathrm{i} S_{\mathrm{CS}}}
$$

This invariant was later mathematically rigorously defined by Reshetikhin and Turaev [54], and it is called the Witten-Reshetikhin-Turaev (WRT) invariant. Simpler construction was given later in Ref. 41 based on the Conway-Kauffman skein relation (see also Refs. 31, 42 ).

Not only the invariant of the 3-manifold $\mathcal{M}$, the Chern-Simons action (2.6) gives the quantum invariant for link $\mathcal{L}$ in $\mathcal{M}$. We define the Wilson loop expectation value by

$$
W_{R_{1}, \ldots, R_{L}}(\mathcal{L})=\frac{1}{Z(\mathcal{M})} \int \mathcal{D} A W_{R_{1}}^{\mathcal{K}_{1}}(A) \cdots W_{R_{L}}^{\mathcal{K}_{L}}(A) \mathrm{e}^{\mathrm{i} S_{\mathrm{CS}}}
$$

where we assume the link $\mathcal{L}$ has components $\mathcal{K}_{1}, \ldots \mathcal{K}_{L}$, and $W_{R}^{\mathcal{K}}$ denotes the Wilson loop operator along a component $\mathcal{K}$ with a representation $R$ of gauge group $G$;

$$
W_{R}^{\mathcal{K}}(A)=\operatorname{Tr}_{R} \mathcal{P} \exp \oint_{\mathcal{K}} A
$$

where $\mathcal{P}$ is a path ordering. When the gauge group $G=S U(2)$, this correlation function becomes the colored Jones polynomial at the root of unity.

\subsection{Combinatorial Construction of $S U(2)$ Quantum Invariants}

Hereafter in this article we only study a case of the gauge group $G=S U(2)$. In $S U(2)$ case, as a $S$-transformation of the conformal block, we use [22]

$$
S_{a b}=\sqrt{\frac{2}{K+2}} \sin \left(\frac{(a+1)(b+1) \pi}{K+2}\right)
$$

In actual computation of the quantum invariants such as $Z(\mathcal{M})$ and $W_{R}(\mathcal{K})$, useful and the most elementary method, at least to the author, is to use the skein theory. Here we briefly review a skein-theoretical construction of the $S U(2)$ quantum invariant (see, e.g., Ref. 31, 42). 
The Jones polynomial is characterized by the skein relation;

$$
\begin{gathered}
\searrow=A)\left(+A^{-1} \smile\right. \\
D \cup \bigcirc=d D
\end{gathered}
$$

Here $A$ is a parameter related to the quantum group deformation parameter $q=A^{4}$, and

$$
d=-A^{2}-A^{-2}
$$

Projection of knots and links is denoted by $D$.

In constructing the colored Jones polynomial of links and the SU(2) WRT invariant of 3manifolds, fundamental tool is the Jones-Wenzl idempotent. Throughout this article, we employ a standard notation in diagrams $D$; an integer $n$ beside an arc means that there exist $n$ copies of that arc. Like (2.11) an arc without integer denotes a single arc, i.e., $n=1$ is often omitted. Then the Jones-Wenzl idempotent is depicted as a blank square, and it may also be recursively defined as

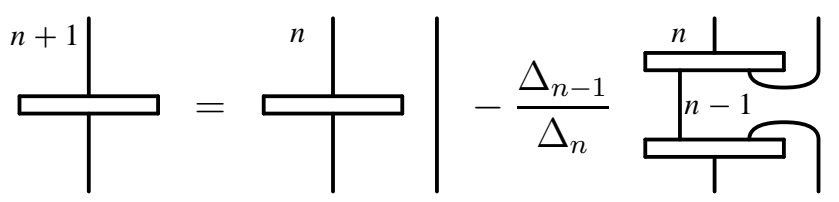

Here $\Delta_{n}$ is the colored Jones polynomial for unknot;

$$
\begin{aligned}
\Delta_{n} & =\overbrace{}^{n} \frac{A^{2 n+2}-A^{-2 n-2}}{A^{2}-A^{-2}}
\end{aligned}
$$

We have $\Delta_{0}=1, \Delta_{1}=d$, and others are recursively defined by

$$
\Delta_{n+1}=d \Delta_{n}-\Delta_{n-1}
$$

Note that we have

$$
\Delta_{x+y+z+1} \Delta_{z-1}=\Delta_{x+z} \Delta_{y+z}-\Delta_{y} \Delta_{x}
$$

One notices that the Jones-Wenzl idempotent satisfies followings;

$$
\begin{aligned}
& \underset{n-m}{\prod_{m} \mid \frac{n}{n}}=\stackrel{n}{n} \\
& \frac{n+2 \mid}{\left.\Upsilon_{m}^{n}\right|_{n-m}}=0
\end{aligned}
$$

where $n \geq m$. 
In the $S U(2)_{K}$ theory, we have $\Delta_{n} \neq 0$ for $1 \leq n \leq K$, and $\Delta_{K+1}=0$; namely the parameter $A$ satisfies

$$
A^{4(K+2)}=1
$$

This condition means that integer $n$ attached to each arc should be $1 \leq n \leq K$. Here we choose [15]

$$
A=\mathrm{i} \exp \left(\frac{\pi \mathrm{i}}{2(K+2)}\right)
$$

from physical requirement which will be explained below. Under this condition we have

$$
\Delta_{n}=\frac{\sin \left(\frac{n+1}{K+2} \pi\right)}{\sin \left(\frac{1}{K+2} \pi\right)}
$$

and especially we have

$$
d=\Delta_{1}=2 \cos \left(\frac{\pi}{K+2}\right)
$$

We introduce a trivalent vertex by

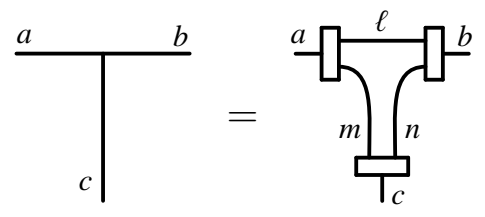

where

$$
\ell=\frac{a+b-c}{2} \quad m=\frac{c+a-b}{2} \quad n=\frac{b+c-a}{2}
$$

Indices in trivalent vertex (2.23) should fulfill an admissible condition;

$$
\left\{\begin{array}{l}
a+b+c=0 \quad \bmod 2 \\
a+b \geq c, \quad b+c \geq a, \quad c+a \geq b \\
a+b+c \leq 2 K
\end{array}\right.
$$

Skein relation (2.11) gives

$$
O=-A^{3}
$$

Generally we have a twist formula;

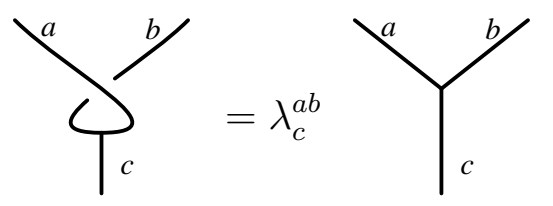

where

$$
\lambda_{c}^{a b}=(-1)^{\frac{a+b-c}{2}} A^{a+b-c+\frac{a^{2}+b^{2}-c^{2}}{2}}
$$




\subsection{Trivalent Diagram as Wilson Line of Quasi-Particles}

The diagram introduced above can be interpreted physically as follows. We regard a single arc as a Wilson line of quasi-particle with spin-1/2. The Jones-Wenzl idempotent which has index $n$ means the projection to spin- $n / 2$ space, and the arc labelled by $n$ corresponds to the Wilson line of quasi-particle with spin- $n / 2$;

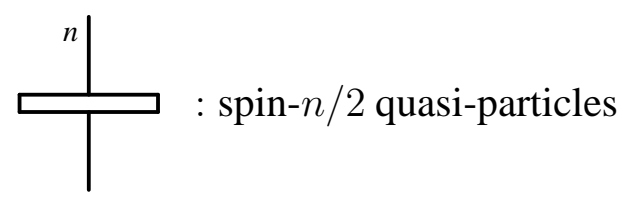

Then the trivalent vertex (2.23) denotes the usual fusion rule; quasi-particles, $\phi_{a}$ and $\phi_{b}$, with spin$a / 2$ and spin- $b / 2$ fuse to spin- $c / 2$ quasi-particle $\phi_{c}$. One sees that the admissible condition (2.24) of the $S U(2)_{K} \mathrm{CS}$ theory has a correspondence with the fusion channel (2.2) [56]. In general the fusion rule is written as

$$
\phi_{a} \times \phi_{b}=\sum_{c} N_{a b}^{c} \phi_{c}
$$

Verlinde formula [57] shows that the the fusion multiplicity can be written in terms of the $S$-matrix as

$$
N_{a b c}=\sum_{d} \frac{S_{a d} S_{b d} S_{c d}}{S_{0 d}}
$$

We recall that the $S$-matrix for $S U(2)$ is given in (2.10). The quantum dimension $d_{a}$ of quasiparticle with spin $a / 2$ is the largest eigenvalue of the matrix $N_{a}$, and in the $S U(2)_{K}$ case we have

$$
d_{a}=\frac{S_{0 a}}{S_{00}}=\Delta_{a}
$$

The non-vanishing correlation function of quasi-particles should fuse into the vacuum section. For example, the correlation function of two quasi-particles with spin- $1 / 2$ will vanish unless two fuse into the vacuum. Diagrammatically we denote this 2-quasi-particle state by

$$
|\widetilde{\mathrm{qp}}\rangle=\circlearrowright
$$

Two endpoints of arc indicate existence of quasi-particle at spatially different points, and a connecting arc denotes that they fuse to the vacuum sector. Naturally a dual state is defined upside down as

$$
\langle\widetilde{\mathrm{qp}}|=\bigcap
$$

We can compute norm of the state by connecting two endpoints of $\langle\widetilde{q p}|$ and $|\widetilde{q p}\rangle$, and (2.12) gives

$$
\langle\widetilde{\mathrm{qp}} \mid \widetilde{\mathrm{qp}}\rangle=\bigcirc=d
$$

As we have set a parameter $A$ as (2.20), we have $d>0$ as a level $K$ is $K \geq 1$. Then we have the normalized physical state, which is schematically written as

$$
|\mathrm{qp}\rangle=\frac{1}{\sqrt{d}} \circlearrowright
$$




\section{TOPOLOGiCAL ENTANGLEMENT ENTROPY}

Entanglement is one of distinguishing properties of quantum mechanics, and it receives much interests in recent studies of quantum information science. One of measures of entanglement is the entropy (see, e.g., Refs. 1 for recent review). Namely when we assume that the state is a pure bipartite state $|\Psi\rangle$, and that the system is divided into two sub-systems $A$ and $B$, the Schmidt decomposition assures that we can write

$$
|\Psi\rangle=\sum_{j} p_{j}\left|\psi_{j}\right\rangle_{A} \otimes\left|\phi_{j}\right\rangle_{B}
$$

where $\left|\psi_{j}\right\rangle_{A}$ and $\left|\phi_{j}\right\rangle_{B}$ are orthonormal states in subspace $A$ and $B$,

$$
{ }_{A}\left\langle\psi_{j} \mid \psi_{k}\right\rangle_{A}=\delta_{j, k} \quad{ }_{B}\left\langle\phi_{j} \mid \phi_{k}\right\rangle_{B}=\delta_{j, k}
$$

and $\sum_{j}\left|p_{j}\right|^{2}=1$. Then the entanglement entropy is defined by

$$
S_{A}=-\sum_{j}\left|p_{j}\right|^{2} \log \left|p_{j}\right|^{2}
$$

We note that

$$
S_{A}=S_{B}
$$

Alternatively the entanglement entropy is defined as the von Neumann entropy

$$
S_{A}=-\operatorname{Tr}_{A}\left(\rho_{A} \log \rho_{A}\right)
$$

Here $\rho_{A}$ is the reduced density matrix (Alice's density matrix)

$$
\rho_{A}=\operatorname{Tr}_{B} \rho=\operatorname{Tr}_{B}(|\Psi\rangle\langle\Psi|)
$$

where the density matrix $\rho$ is

$$
\rho=|\Psi\rangle\langle\Psi|
$$

In fact when we use the replica trick [6, 26]

$$
S_{A}=-\lim _{n \rightarrow 1} \frac{\partial}{\partial n}\left(\operatorname{Tr}_{A} \rho_{A}^{n}\right)
$$

we obtain (3.3).

In the non-Abelian states, it is expected that there exists an effect from a topological order [35, 39]. Those papers discuss that such effect appears from a dependence on a length of boundary between $A$ and $B$. As our model is purely topological and pure gauge theory, we rather define the topological entanglement entropy by

$$
S_{A}^{\text {topo }}=-\operatorname{Tr}_{A}\left(\rho_{A} \log \rho_{A}\right)+\sum_{j}\left|p_{j}\right|^{2} \log \left|p_{j}\right|^{2}
$$

We shall compute the topological entanglement entropy (3.9) of the non-Abelian quasi-particle states in the following. 
We explain how to compute the topological entanglement based on the skein theory. In our formulation, the state is schematically written as

$$
|\Psi\rangle=211
$$

where each end of arcs denotes quasi-particles at spatially different points. There exists a sum of trivalent graphs insides an ellipse, which is a Wilson line describing how quasi-particles fuse to the vacuum sector. Correspondingly the density matrix (3.7) is depicted as

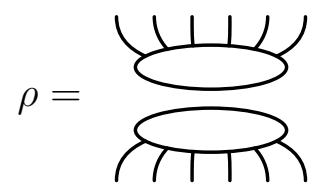

We now divide quasi-particles into two groups $A$ and $B$. We mean that the owner of each quasiparticle is Alice or Bob. As the Alice's reduced density matrix $\rho_{A}(3.6)$ is given by taking traces in Bob's space, it is depicted as follows by connecting ends of Bob's arcs;

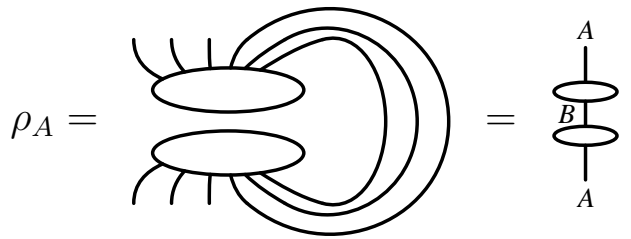

where in the last expression, $A$ and $B$ denote quasi-particles belonging to Alice and Bob respectively, not spins of quasi-particles. Applying (3.8), the von Neumann entropy (3.5) is computed by connecting $n$ copies of the reduced density matrix $\rho_{A}$;

$$
S_{A}=-\lim _{n \rightarrow 1} \frac{\partial}{\partial n} \sum_{A}^{A}\left[\sum_{A}^{A}: \sum_{A}^{A}\right.
$$

Above trivalent graphs are computable by the skein theory, and we can obtain explicitly the topological entanglement entropy from (3.9).

\section{QUASI-PARTICLES WITH SPIN $1 / 2$}

We study the braiding operations on the correlation function of many-quasi-particles with spin1/2. The Bratteli diagram of spin-1/2 quasi-particles is depicted in Fig. 1. One sees that the number of quasi-particles must be even so that the correlation function is non-vanishing. In the case of 2-quasi-particle state, we have only a unique path which starts from $(0,0)$ and ends at $(2,0)$ in the Bratteli diagram in Fig. 1, which corresponds to the state (2.34). Generally we have several paths from $(0,0)$ to $(2 n, 0)$. The number of paths denotes that of the fusion channels, and it is the dimension of the Hilbert space of $2 n$-quasi-particle states. We see that, for the $S U(2)_{2}$ theory, it is $2^{n-1}$, which denotes the dimension studied in Ref. 46. 


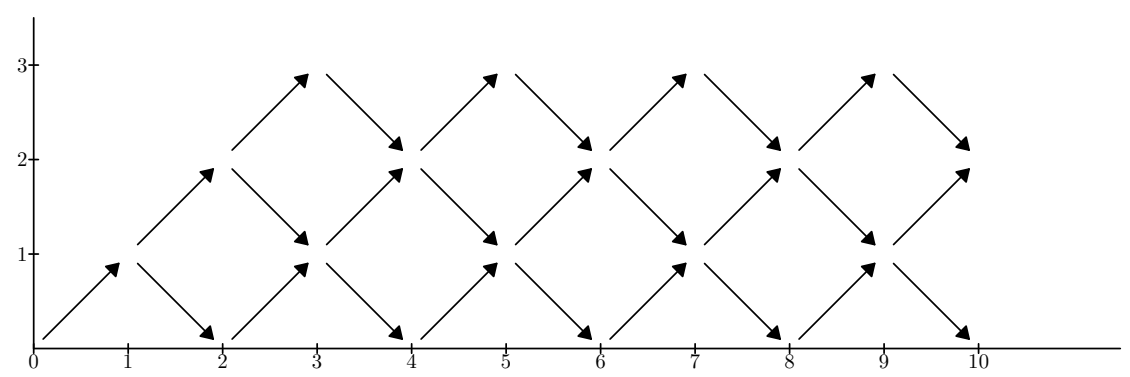

Figure 1: Bratteli diagram for spin-1/2 for $S U(2)_{3}$ theory.

We shall study the action of the braid operators on the $2 n$-quasi-particle states. The nonAbelian property of quasi-particles is that we have the nontrivial representation of the braid operators. Hereafter $\sigma_{i}$ means the braid operator acting on the $i$-th and $i+1$-th particles as

$$
\sigma_{i}=|\cdots \cdots \cdots \cdots . . . .| \sum_{i+1}|\ldots \ldots \ldots \ldots . . .|
$$

Here $i$ and $i+1$ mean the $i$-th and $i+1$-th positions from the left respectively, and these should not be confused with spins of quasi-particles. The operators $\sigma_{i}$ satisfy the Artin braid relation

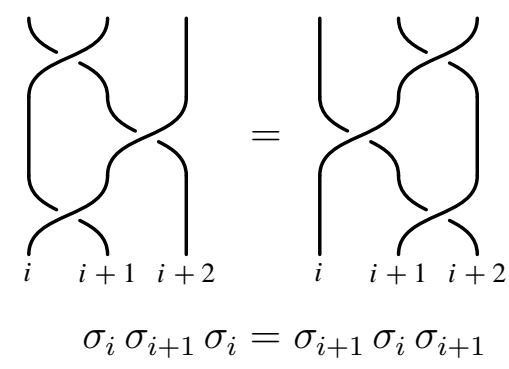

Easy is to see that

$$
\sigma_{i} \sigma_{j}=\sigma_{j} \sigma_{i}
$$

for $|i-j| \geq 2$. We also use the twist $\theta_{i}$;

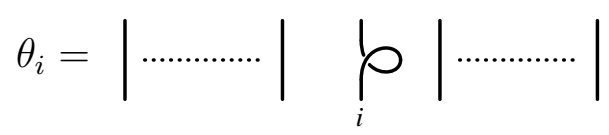

\subsection{4-Quasi-Particle States}

We study the braiding effects in the 4-quasi-particle states in the $S U(2)_{K \geq 2}$ theory. A dimension of the Hilbert space of 4 quasi-particles is two, and we may choose unnormalized bases as

$$
\begin{aligned}
& |\widetilde{0}\rangle=\bigcup \mho \\
& |\widetilde{1}\rangle=\bigcup_{2} \bigcup
\end{aligned}
$$


Correspondingly dual bases $\langle\widetilde{0}|$ and $\langle\widetilde{1}|$ are defined upside down. The norms of these diagrams are computed as

$$
\begin{aligned}
& \langle\widetilde{0} \mid \widetilde{0}\rangle=\bigcirc \bigcirc=d^{2} \\
& \langle\widetilde{1} \mid \tilde{1}\rangle=\overbrace{2}^{2}=\overbrace{}^{2}=\Delta_{2}
\end{aligned}
$$

where we have used

$$
\bigcap_{21}^{21}=2^{2}
$$

We see that they have positive norms, $d^{2}>0$ and $\Delta_{2}>0$, under a parametrization (2.20). It is noted that, when $K=1$, we have $\Delta_{2}=0$, and that $|\tilde{1}\rangle$ becomes a null state due to that it does not satisfy the admissible condition (2.24). We also see that

$$
\langle\widetilde{0} \mid \widetilde{1}\rangle=\underbrace{}_{2} \bigcup=0
$$

by making use of the following identity, which is a special case of (2.18);

$$
\bigcap_{2}=0
$$

Thus we indeed have two normalized physical states

$$
\begin{gathered}
|0\rangle=\frac{1}{d} \cup \mho \\
|1\rangle=\frac{1}{\sqrt{d^{2}-1}} \bigcup_{2} \bigcup
\end{gathered}
$$

which are orthonormal; for $i . j \in\{0,1\}$ we have

$$
\langle i \mid j\rangle=\delta_{i, j}
$$

We study actions of braid operators on states $\{|0\rangle,|1\rangle\}$. Here we use the $F$-matrix defined by

$$
\left(\begin{array}{c}
)( \\
)^{2}(
\end{array}\right)=\mathbf{F}_{11}^{11}\left(\begin{array}{l}
\underset{\tau}{I_{2}} \\
\overbrace{}^{2}
\end{array}\right)
$$

We see that

$$
\mathbf{F}_{11}^{11}=\left(\begin{array}{cc}
\frac{1}{d} & 1 \\
1-\frac{1}{d^{2}} & -\frac{1}{d}
\end{array}\right)
$$

which can be computed by use of a recursion relation of the Jones-Wenzl idempotent (2.14). Actions of $\sigma_{1}$ and $\sigma_{3}$ can be computed easily by use of (2.25). For instance, we have

$$
\sigma_{1} \cup \cup=\bigcup \mid \mathcal{U}^{-3} \cup \cup
$$


To get an action of $\sigma_{2}$, the $F$-matrix (4.8) will be useful. We have

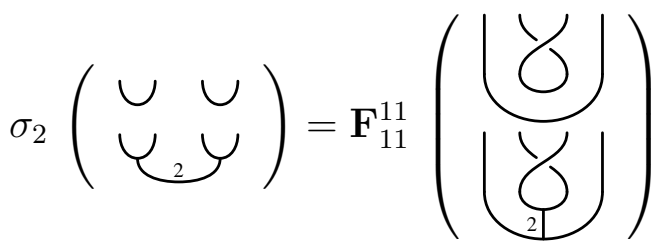

$$
\begin{aligned}
& =\mathbf{F}_{11}^{11}\left(\begin{array}{cc}
-A^{3} & 0 \\
0 & A
\end{array}\right)(\underset{\smile}{\smile}) \\
& =\mathbf{F}_{11}^{11}\left(\begin{array}{cc}
-A^{3} & 0 \\
0 & A
\end{array}\right)\left(\mathbf{F}_{11}^{11}\right)^{-1}\left(\begin{array}{ll}
\cup & \cup \\
\bigcup_{2} & \mho
\end{array}\right)
\end{aligned}
$$

Actions of other operators can be computed in the same way. In summary, the representation of the braid operators on the space spanned by $\{|0\rangle,|1\rangle\}$ (4.6) are as follows;

$$
\begin{gathered}
\rho\left(\sigma_{1}\right)=\rho\left(\sigma_{3}\right)=\left(\begin{array}{ll}
-A^{-3} & \\
& A
\end{array}\right) \\
\rho\left(\sigma_{2}\right)=\left(\begin{array}{cc}
-\frac{A^{3}}{d} & \frac{\sqrt{d^{2}-1}}{A d} \\
\frac{\sqrt{d^{2}-1}}{A d} & \frac{1}{A^{5} d}
\end{array}\right)
\end{gathered}
$$

which are unitary under (2.20). We can check directly that these representations satisfy the Artin braid relation (4.2), and that

$$
\rho\left(\sigma_{1} \sigma_{2} \sigma_{3}^{2} \sigma_{2} \sigma_{1}\right)=A^{-6} \cdot \mathbf{1}
$$

Computation of the representation of the twist (4.3) is straightforward. As all quasi-particles are spin- $1 / 2$, the representation of $\theta_{i}$ is same for all $i$, and we have

$$
\rho(\theta)=-A^{3}
$$

It is remarked that these representations are computed in Ref. 2 from an explicit form of wave functions as the 4-point correlation functions of the WZW model [38].

\subsection{6-Quasi-Particle States}

We next study 6-quasi-particle states. We restrict to the case $K>2$. The $S U(2)_{2}$ case will be discussed separately in the later section. As can be seen from the Bratteli diagram in Fig. 1, the 
dimension of the Hilbert spaces is 5 . We set

$$
\begin{aligned}
& |00\rangle=\frac{1}{d \sqrt{d}} \cup \cup \mho \\
& |01\rangle=\frac{1}{\sqrt{d\left(d^{2}-1\right)}} \cup \mho_{2} \\
& |10\rangle=\frac{1}{\sqrt{d\left(d^{2}-1\right)}} \bigcup_{2} \bigcup \mho \\
& |11\rangle=\frac{1}{\sqrt{d\left(d^{2}-1\right)}} \bigcup \bigcup_{2} Y \\
& |C\rangle=\sqrt{\frac{d}{\left(d^{2}-1\right)\left(d^{2}-2\right)}} \bigcup_{2} Y_{2} Y
\end{aligned}
$$

We have defined these states based on Ref. 20. See that (2.20) proves positivity of norm of each diagram, and by the same computation with a case of 4-quasi-particle, we see that these are orthonormal bases

$$
\langle i \mid j\rangle=\delta_{i, j}
$$

where $i, j \in\{00,01,10,11, C\}$.

We study the action of the braid operators $\left\{\sigma_{1}, \ldots, \sigma_{5}\right\}$. Actions of $\sigma_{1}, \sigma_{3}$, and $\sigma_{5}$ are easily computed by use of (2.26). Actions of $\sigma_{2}$ on $|00\rangle$ and $|10\rangle$, and of $\sigma_{4}$ on $|00\rangle$ and $|01\rangle$, can be given in the same method with 4-quasi-particles. For the action of others, we may use

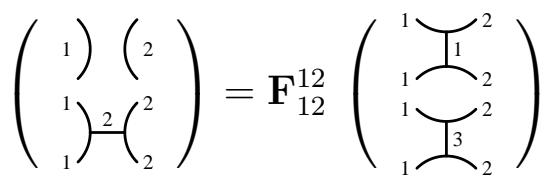

Here the $F$-matrix is given by

$$
\mathbf{F}_{12}^{12}=\left(\begin{array}{cc}
\frac{d}{d^{2}-1} & 1 \\
1-\frac{1}{d^{2}-1} & -\frac{1}{d}
\end{array}\right)
$$

which is derived by the recursion relation of the Jones-Wenzl idempotent (2.14). Then, for example, we compute as follows;

$$
\begin{aligned}
& \sigma_{2} \cup Y_{2} Y=U \underbrace{}_{2} \\
& =\left(\mathbf{F}_{11}^{11}\right)_{11} \bigcirc \mathrm{O}_{2}+\left(\mathbf{F}_{11}^{11}\right)_{11}\left(\mathbf{S}_{2}\right. \\
& =\left(\mathbf{F}_{11}^{11}\right)_{11}\left(-A^{-3}\right) \smile \mathcal{L}_{2} Y+\left(\mathbf{F}_{11}^{11}\right)_{11} A \cup \mathcal{L}_{2} Y \\
& =\left(\mathbf{F}_{11}^{11}\left(\begin{array}{cc}
-A^{-3} & 0 \\
0 & A
\end{array}\right)\left(\mathbf{F}_{11}^{11}\right)^{-1}\right)_{11} \cup \bigcup_{2} Y \\
& +\left(\mathbf{F}_{11}^{11}\left(\begin{array}{cc}
-A^{-3} & 0 \\
0 & A
\end{array}\right)\left(\mathbf{F}_{11}^{11}\right)^{-1}\right)_{12} Y_{2} Y_{2} \mathcal{Y}
\end{aligned}
$$


In the last expression, we further apply (4.13) to get

$$
Y_{2} Y_{2} \mho=\left(\left(\mathbf{F}_{12}^{12}\right)^{-1}\right)_{11} Y \cup_{2} Y+\left(\left(\mathbf{F}_{12}^{12}\right)^{-1}\right)_{12} Y_{2} Y_{12} Y
$$

Other actions can be computed in the similar methods.

To summarize, the representation of the braid operators on the 5-dimensional space $\{|00\rangle,|01\rangle,|10\rangle,|11\rangle,|C\rangle\}$ are given by

$$
\begin{gathered}
\rho\left(\sigma_{2}\right)=\left(\begin{array}{ccccc}
-\frac{A^{3}}{d} & 0 & \frac{\sqrt{d^{2}-1}}{A d} & 0 & 0 \\
0 & -\frac{A^{3}}{d} & 0 & \frac{1}{A d} & \frac{\sqrt{d^{2}-2}}{A d} \\
\frac{\sqrt{d^{2}-1}}{A d} & 0 & \frac{1}{A^{5} d} & 0 & 0 \\
0 & \frac{1}{A d} & 0 & -\frac{A^{3}}{d} & \frac{\sqrt{d^{2}-2}}{A d} \\
0 & \frac{\sqrt{d^{2}-2}}{A d} & 0 & \frac{\sqrt{d^{2}-2}}{A d} & A+\frac{d^{2}-2}{A d}
\end{array}\right) \\
\rho\left(\sigma_{4}\right)= \\
\rho\left(\sigma_{3}\right)=\operatorname{diag}\left(-A^{-3}, A, A,-A^{-3}, A\right) \\
\left.\begin{array}{ccccc}
-\frac{A^{3}}{d} & \frac{\sqrt{d^{2}-1}}{A d} & 0 & 0 & 0 \\
\frac{\sqrt{d^{2}-1}}{A d} & \frac{1}{A^{5} d} & 0 & 0 & 0 \\
0 & 0 & -\frac{A^{3}}{d} & \frac{1}{A d} & \frac{\sqrt{d^{2}-2}}{A d} \\
0 & 0 & \frac{1}{A d} & -\frac{A^{3}}{d} & \frac{\sqrt{d^{2}-2}}{A d} \\
0 & 0 & \frac{\sqrt{d^{2}-2}}{A d} & \frac{\sqrt{d^{2}-2}}{A d} & A+\frac{d^{2}-2}{A d}
\end{array}\right) \\
\rho\left(\sigma_{5}\right)=\operatorname{diag}\left(-A^{-3}, A,-A^{-3}, A, A\right)
\end{gathered}
$$

One can check that these satisfy (4.2), and

$$
\rho\left(\sigma_{1} \sigma_{2} \sigma_{3} \sigma_{4} \sigma_{5} \sigma_{5} \sigma_{4} \sigma_{3} \sigma_{2} \sigma_{1}\right)=A^{-6} \cdot \mathbf{1}
$$

Note that the representation of the twist (4.3) is same with before, and we have (4.11).

\subsection{Topological Entanglement Entropy of Spin-1/2 Quasi-Particle States}

\section{过 2-Quasi-Particle State}

As the first simple example of explicit computation of entanglement entropy, we study the 2quasi-particle state (2.34). We have the state $|\Psi\rangle=|\mathrm{qp}\rangle$, and the density matrix $\rho$ is depicted as

$$
\rho=\frac{1}{d} \bigcirc
$$

We assume that both Alice and Bob have one quasi-particle. Alice (resp. Bob) has the left (resp. right) quasi-particle, and we denote it as $A=\{1\}$ and $B=\{2\}$. As a trace $\operatorname{Tr}_{B}$ means connecting 
arc ends of Bob's quasi-particles (3.12), we have

$$
\rho_{\{1\}}=\operatorname{Tr}_{\{2\}} \rho=\frac{1}{d}
$$

Then from (3.13) we obtain the entanglement entropy as

$$
S_{\{1\}}=-\lim _{n \rightarrow 1} \frac{\partial}{\partial n}\left(\frac{1}{d^{n}} \bigcirc\right)=\log d
$$

and the topological entanglement entropy (3.9) is identified with

$$
S_{\{1\}}^{\text {topo }}=S_{\{1\}}=\log d
$$

This result is due to that Alice and Bob are intertwined by a Wilson line of spin- $1 / 2$ quasi-particle whose quantum dimension is $d(2.30)$.

\section{度 4-Quasi-Particle States}

We next consider the 4-quasi-particle states (4.6), which spans two-dimensional space. We take a state as

$$
|\Psi\rangle=p_{0}|0\rangle+p_{1}|1\rangle
$$

where $\left|p_{0}\right|+\left|p_{1}\right|^{2}=1$. We first assume that the first and the second quasi-particles from the left belong to Alice, and that the remaining third and fourth quasi-particles to Bob; we denote $A=\{1,2\}$ and $B=\{3,4\}$. Connecting ends of Wilson lines of Bob's quasi-particles as in (3.12) and using (4.4) and (4.5), Alice's reduced density matrix is schematically written as

$$
\rho_{\{1,2\}}=\left|p_{0}\right|^{2} \frac{1}{d} \bigcap^{2}+\left|p_{1}\right|^{2} \frac{1}{d^{2}-1} \bigcap_{2}
$$

It is straightforward to compute (3.13), and we get the von Neumann entropy as

$$
\begin{aligned}
S_{\{1,2\}} & \left.=-\lim _{n \rightarrow 1} \frac{\partial}{\partial n}\left(\left(\frac{\left|p_{0}\right|^{2}}{d}\right)^{n}(\bigcirc)^{n}+\left(\frac{\left|p_{1}\right|^{2}}{d^{2}-1}\right)^{n} \bigcirc 2\right)\right) \\
& =-\left|p_{0}\right|^{2} \log \left|p_{0}\right|^{2}-\left|p_{1}\right|^{2} \log \left(\frac{\left|p_{1}\right|^{2}}{d^{2}-1}\right)
\end{aligned}
$$

where we have used (4.4) and (4.5). To extract a topological entropy, we notice that the state (4.20) has the orthogonal bases $\left|\psi_{j}\right\rangle_{\{1,2\}}$ and $\left|\phi_{j}\right\rangle_{\{3,4\}}$ as (3.2) thanks to (4.4) and (4.5). As a result, the topological entanglement entropy (3.9) is identified with

$$
S_{\{1,2\}}^{\text {topo }}=\left|p_{1}\right|^{2} \log \left(d^{2}-1\right)
$$

This result is interpreted as follows. The state $|0\rangle$ is not topologically entangled between Alice and Bob, or they are entangled by the vacuum sector with quantum dimension $d_{0}=1$. Although, in the state $|1\rangle$ Alice's quasi-particles are intertwined with Bob's through the Wilson line of quasiparticle with spin-1 whose quantum dimension (2.30) is $d_{2}=\Delta_{2}=d^{2}-1$. 
We make similar computations for the 4-quasi-particle states (4.6) when Alice owns the second and the third particles while Bob has remainder; $A=\{2,3\}$ and $B=\{1,4\}$. Following (3.12) the reduced density matrix is given by

$$
\begin{aligned}
\rho_{\{2,3\}} & =\frac{\left|p_{0}\right|^{2}}{d^{2}}\left|+\frac{p_{0} p_{1}^{*}+p_{0}^{*} p_{1}}{d \sqrt{d^{2}-1}}\right| 2 \mid+\frac{\left|p_{1}\right|^{2}}{d^{2}-1} \bigcup_{2}^{2} \\
& =\frac{\left|\widetilde{p_{1}}\right|^{2}}{d^{2}-1} \stackrel{2}{ }+\frac{\left|\widetilde{p_{0}}\right|^{2}}{d} \circlearrowright
\end{aligned}
$$

where

$$
\begin{aligned}
& \widetilde{p_{0}}=\frac{p_{0}}{d}+p_{1} \frac{\sqrt{d^{2}-1}}{d} \\
& \widetilde{p_{1}}=p_{0} \frac{\sqrt{d^{2}-1}}{d}-\frac{p_{1}}{d}
\end{aligned}
$$

Here, in the second equality, we have substituted a definition of a trivalent vertex (2.23) and have used the Jones-Wenzl idempotent (2.14). Note that $\left|\widetilde{p_{0}}\right|^{2}+\left|\widetilde{p_{1}}\right|^{2}=1$. Using (2.18) the entanglement entropy (3.13) is computed as

$$
\begin{aligned}
S_{\{2,3\}} & =-\lim _{n \rightarrow 1} \frac{\partial}{\partial n}\left(\left(\frac{\left|\widetilde{p_{1}}\right|^{2}}{d^{2}-1}\right)^{n} \bigcirc 2+\left(\frac{\left|\widetilde{p_{0}}\right|^{2}}{d}\right)^{n}(\bigcirc)^{n}\right) \\
& =-\left|\widetilde{p_{1}}\right|^{2} \log \left(\frac{\left|\widetilde{p_{1}}\right|^{2}}{d^{2}-1}\right)-\left|\widetilde{p_{0}}\right|^{2} \log \left|\widetilde{p_{0}}\right|^{2}
\end{aligned}
$$

To extract the topological entanglement entropy, we should note that bases in (4.6) do not give orthogonal bases, $\left|\psi_{j}\right\rangle_{\{2,3\}}$ and $\left|\phi_{j}\right\rangle_{\{1,4\}}$, in Alice's and Bob's spaces (3.2). To remedy it we rewrite the state (4.20) by use of the $F$-matrix (4.8) as

$$
|\Psi\rangle=\widetilde{p_{0}} \frac{1}{d} \circlearrowright+\widetilde{p_{1}} \frac{1}{\sqrt{d^{2}-1}} \circlearrowright 2
$$

which has a form of (3.2), i.e., the states $\left|\psi_{j}\right\rangle_{\{2,3\}}$ and $\left|\phi_{j}\right\rangle_{\{1,4\}}$ are orthonormal due to (4.4) and (4.5) (see, e.g., (7.8)). Thus the topological entanglement entropy (3.5) is given by

$$
S_{\{2,3\}}^{\text {topo }}=\left|\widetilde{p_{1}}\right|^{2} \log \left(d^{2}-1\right)
$$

When we look at the expression (4.27), we see that Alice's and Bob's quasi-particles are not topologically entangled with a probability $\left|\widetilde{p_{0}}\right|^{2}$ while they are intertwined with a probability $\left|\widetilde{p_{1}}\right|^{2}$ by the Wilson line of quasi-particle with spin- 1 whose quantum dimension is $d_{2}=d^{2}-1$. Thus the expression (4.27) is comparable with (4.20) replacing $p_{a}$ with $\widetilde{p_{a}}$.

We leave it for readers to check that

$$
S_{\{1\}}^{\mathrm{topo}}=S_{\{2,3,4\}}^{\mathrm{topo}}=\log d
$$

which supports our interpretation because Alice and Bob are intertwined by spin- $1 / 2$ quasiparticle (consult computations in Section 7.4). In view of (4.23), (4.28), and (4.29), the topological entanglement entropy depends on what quasi-particles Alice and Bob have in the state $|\Psi\rangle$. Even 
if the state is same, there exists a possibility that a different quantum dimension appears depending on a quasi-particle which intertwines Alice and Bob when we change owners of quasi-particles.

\subsection{Pfaffian State}

The Moore-Read Pfaffian state is described by the $S U(2)_{2}$ CS theory, and we have $A=\mathrm{i} \mathrm{e}^{\pi \mathrm{i} / 8}$ and $d=\sqrt{2}$. We then have a representation of the twist $\theta_{i}(4.3)$ as

$$
\rho(\theta)=-\mathrm{e}^{-\pi \mathrm{i} / 8}
$$

The braid matrices (4.10) for 4-quasi-particle states (4.6) become

$$
\begin{gathered}
\rho\left(\sigma_{1}\right)=\rho\left(\sigma_{3}\right)=\mathrm{e}^{\pi \mathrm{i} / 8}\left(\begin{array}{ll}
-1 & \\
& \mathrm{i}
\end{array}\right) \\
\rho\left(\sigma_{2}\right)=-\frac{\mathrm{e}^{-\frac{1}{8} \pi \mathrm{i}}}{\sqrt{2}}\left(\begin{array}{ll}
1 & \mathrm{i} \\
\mathrm{i} & 1
\end{array}\right)
\end{gathered}
$$

For 6-quasi-particle, the state $|C\rangle$ amongst (4.12) is not admissible due to (2.24); we can see that norm of the diagram for $|C\rangle$ reduces to zero, and it is unphysical, null state. Then from (4.15) the braiding matrices on space spanned by bases $\{|00\rangle,|01\rangle,|10\rangle,|11\rangle\}$ in (4.12) are given by

$$
\begin{gathered}
\rho\left(\sigma_{1}\right)=\mathrm{e}^{\pi \mathrm{i} / 8} \operatorname{diag}(-1,-1, \mathrm{i}, \mathrm{i}) \\
\rho\left(\sigma_{2}\right)=-\frac{\mathrm{e}^{-\frac{1}{8} \pi \mathrm{i}}}{\sqrt{2}}\left(\begin{array}{cccc}
1 & 0 & \mathrm{i} & 0 \\
0 & 1 & 0 & \mathrm{i} \\
\mathrm{i} & 0 & 1 & 0 \\
0 & \mathrm{i} & 0 & 1
\end{array}\right) \\
\rho\left(\sigma_{3}\right)=\mathrm{e}^{\pi \mathrm{i} / 8} \operatorname{diag}(-1, \mathrm{i}, \mathrm{i},-1) \\
\rho\left(\sigma_{4}\right)=-\frac{\mathrm{e}^{-\frac{1}{8} \pi \mathrm{i}}}{\sqrt{2}}\left(\begin{array}{cccc}
1 & \mathrm{i} & 0 & 0 \\
\mathrm{i} & 1 & 0 & 0 \\
0 & 0 & 1 & \mathrm{i} \\
0 & 0 & \mathrm{i} & 1
\end{array}\right) \\
\rho\left(\sigma_{5}\right)=\mathrm{e}^{\pi \mathrm{i} / 8} \operatorname{diag}(-1, \mathrm{i},-1, \mathrm{i})
\end{gathered}
$$

These representations are studied in Ref. 19. Also see Ref. 28 where studied are the braiding matrices of vortices in a $p$-wave superconductor.

Concerning the topological entanglement entropy, we see that the quantum dimensions (2.30) are given by $d_{1}=\sqrt{2}$ and $d_{2}=1$, and that only spin-1/2 quasi-particles contribute to the topological entanglement entropy. Our results, 4.23) and (4.28), indicate that the topological entanglement entropy vanishes in the 4-quasi-particle states (4.20) when both Alice and Bob have two quasi-particles. Note that it does not vanish when Alice has one or three quasi-particles (see 4.29). 


\section{QUASI-PARTICLES WITH SPIN-1}

We study the braid group operations on the correlation functions of quasi-particles with spin-1. The fusion rule of spin-1 gives the Bratteli diagram as in Fig. 2. As in the previous section, the number of paths from $(0,0)$ to $(n, 0)$ is the dimension of the conformal block of $n$-quasi-particles. See that, different from the spin- $1 / 2$ case, we have the non-vanishing correlation function even when $n$ is odd. Both the dimension of 2- and 3-quasi-particle states is one, and the normalized 2-quasi-particle and 3-quasi-particle states are respectively given by

$$
\begin{gathered}
\left|\mathrm{qp}_{2}\right\rangle=\frac{1}{\sqrt{d^{2}-1}} \bigcup_{2} \\
\left.\left|\mathrm{qp}_{3}\right\rangle=\sqrt{\frac{d}{\left(d^{2}-1\right)\left(d^{2}-2\right)}} \bigcup^{2}\right]^{2}
\end{gathered}
$$

In $\left|\mathrm{qp}_{2}\right\rangle$ two endpoints of arc labelled 2 indicates that there are two quasi-particles with spin-1 in spatially different points. Correspondingly in $\left|q p_{3}\right\rangle$ three ends of arcs denote that there are three quasi-particles in spatially different points.

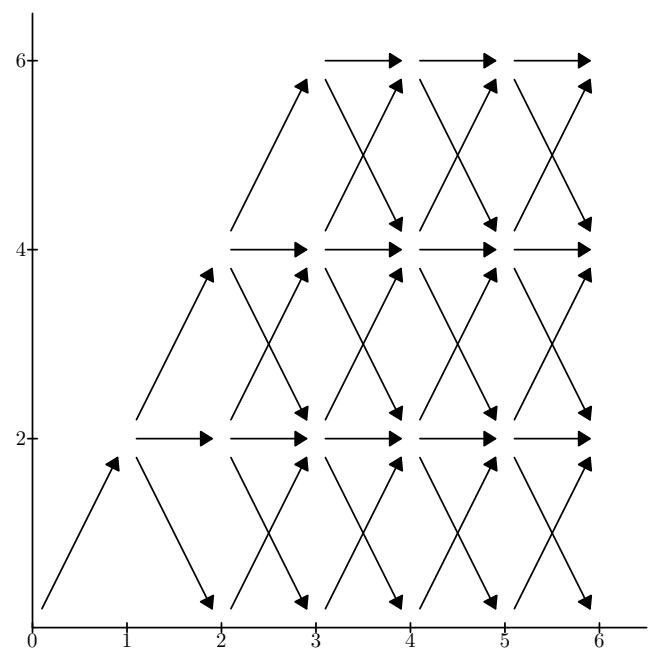

Figure 2: Bratteli diagram for spin-1 for $S U(2)_{7}$ theory

\subsection{4-Quasi-Particles States}

We first assume $K>3$. The $S U(2)_{3}$ theory will be discussed later. A dimension of the Hilbert space of 4-quasi-particles with spin-1 is three as can be read from the Bratteli diagram in Fig. 2 , 
and we set

$$
\begin{gathered}
|0\rangle=\frac{1}{d^{2}-1} \bigcup_{2} \bigcup_{2}^{2} \\
|1\rangle=\frac{d}{\left(d^{2}-2\right) \sqrt{d^{2}-1}} \bigcup_{2}^{2} Y^{2} \\
|2\rangle=\frac{1}{\sqrt{d^{4}-3 d^{2}+1}} Y_{4}^{2}
\end{gathered}
$$

A parameter (2.20) supports positivity of norm of each diagram, and the recursion relation (2.14) of the Jones-Wenzl idempotent proves the orthonormality;

$$
\langle i \mid j\rangle=\delta_{i, j}
$$

The $F$-matrix which we use in this section is

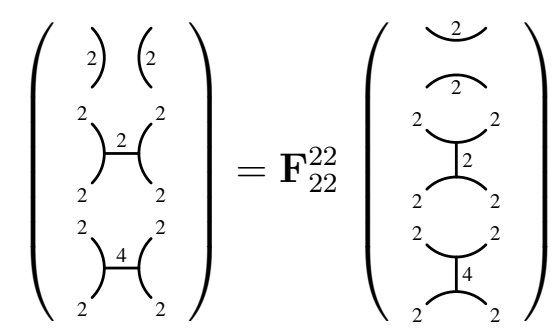

where

This identity follows from

$$
\mathbf{F}_{22}^{22}=\left(\begin{array}{ccc}
\frac{1}{d^{2}-1} & \frac{d}{d^{2}-2} & 1 \\
\frac{d^{2}-2}{d\left(d^{2}-1\right)} & 1-\frac{1}{d^{2}-2} & -\frac{1}{d} \\
1-\frac{d^{2}}{\left(d^{2}-1\right)^{2}} & -\frac{d\left(d^{4}-3 d^{2}+1\right)}{\left(d^{2}-1\right)\left(d^{2}-2\right)^{2}} & \frac{1}{\left(d^{2}-1\right)\left(d^{2}-2\right)}
\end{array}\right)
$$

$$
\begin{aligned}
& \overbrace{2}^{2} I_{2}^{2}={ }_{2}^{2} \overbrace{1}^{1} \gamma_{2}^{2}-\frac{1}{d} \underset{2}{2} \\
& \left.{ }_{2}^{2} I_{4}^{2}=2\right)\left(2-\frac{d^{2}}{\Delta_{3}}{ }_{2}^{2} \partial_{1}^{1} \gamma_{2}^{2}+\frac{d}{\Delta_{2} \Delta_{3}} \underset{2}{\stackrel{2}{2}}\right.
\end{aligned}
$$

which can be derived after some algebra.

Actions of the braid operators on bases $\{|0\rangle,|1\rangle,|2\rangle\}$ (5.3) are computed similarly. Representations of $\sigma_{1}$ and $\sigma_{3}$ can be given from (2.26) straightforwardly, and that of $\sigma_{2}$ can be given once we use the $F$-matrix (5.4). Explicitly they are written as

$$
\begin{gathered}
\rho\left(\sigma_{1}\right)=\rho\left(\sigma_{3}\right)=\operatorname{diag}\left(A^{-8},-A^{-4}, A^{4}\right) \\
\rho\left(\sigma_{2}\right)=\left(\begin{array}{ccc}
\frac{A^{8}}{d^{2}-1} & -\frac{A^{4}}{\sqrt{d^{2}-1}} & \frac{\sqrt{d^{4}-3 d^{2}+1}}{A^{4}\left(d^{2}-1\right)} \\
-\frac{A^{4}}{\sqrt{d^{2}-1}} & 1-\frac{1}{d^{2}-2} & \frac{1}{A^{8}\left(d^{2}-2\right)} \sqrt{\frac{d^{4}-3 d^{2}+1}{d^{2}-1}} \\
\frac{\sqrt{d^{4}-3 d^{2}+1}}{A^{4}\left(d^{2}-1\right)} & \frac{1}{A^{8}\left(d^{2}-2\right)} \sqrt{\frac{d^{4}-3 d^{2}+1}{d^{2}-1}} & \frac{1}{A^{16}\left(d^{2}-1\right)\left(d^{2}-2\right)}
\end{array}\right)
\end{gathered}
$$

See that the braid relation (4.2) is fulfilled, and that

$$
\rho\left(\sigma_{1} \sigma_{2} \sigma_{3} \sigma_{3} \sigma_{2} \sigma_{1}\right)=A^{-16} \cdot \mathbf{1}
$$


The twist (4.3) is follows from (2.26) because all the quasi-particles are spin-1;

$$
\rho(\theta)=A^{8}
$$

\subsection{5-Quasi-Particles States}

In $S U(2)_{K}$ theory with $K \geq 5$, the dimension of the Hilbert space of 5-quasi particles is 6 . We choose bases as

$$
\begin{aligned}
& |01\rangle=\frac{d}{\Delta_{2} \sqrt{\Delta_{3}}} \bigcup^{2} \bigcup^{2} \bigcup^{2} \\
& |10\rangle=\frac{d}{\Delta_{2} \sqrt{\Delta_{3}}} \bigcup^{2} \jmath^{2} \mathcal{U}^{2} \\
& |11\rangle=\left.\frac{d^{3}}{\Delta_{3} \sqrt{\Delta_{2} \Delta_{3}}} \bigcup_{2}^{2}\right|_{2} ^{2} y^{2} \\
& |12\rangle=\left.\frac{d}{\sqrt{\Delta_{3} \Delta_{4}}} \bigcup_{2}^{2}\right|_{4} ^{2} Y^{2} \\
& |21\rangle=\left.\frac{d}{\sqrt{\Delta_{3} \Delta_{4}}} \bigcup_{4}^{2}\right|_{2} ^{2} y^{2} \\
& |22\rangle=\sqrt{\frac{d \Delta_{3}}{\Delta_{4} \Delta_{5}}} \mho_{4}^{2} \int_{4}^{2} y^{2}
\end{aligned}
$$

Here $|a b\rangle$ means that intermediate states have spins- $a, b$ as can be seen from (5.8), and we give generalization later by use of the quantum $6 j$-symbols. We have normalized these states to be $\langle i \mid j\rangle=\delta_{i, j}$, where dual states $\langle i|$ are defined using upside down trivalent graph in (5.8) with the same normalization factors.

To obtain the representation of the braid operators, we need another $F$-matrix beside (5.4);

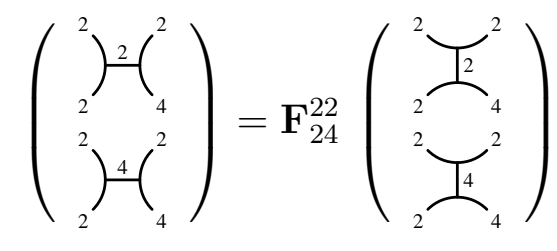

where

$$
\mathbf{F}_{24}^{22}=\left(\begin{array}{cc}
-\frac{d}{\Delta_{3}} & 1 \\
1-\frac{d^{2}}{\left(\Delta_{3}\right)^{2}} & \frac{d}{\Delta_{3}}
\end{array}\right)
$$

A twist formula (2.26) gives representations on spaces spanned by $\{|01\rangle,|10\rangle,|11\rangle,|12\rangle,|21\rangle,|22\rangle\}$;

$$
\begin{aligned}
& \rho\left(\sigma_{1}\right)=\operatorname{diag}\left(A^{-8},-A^{-4},-A^{-4},-A^{-4}, A^{4}, A^{4}\right) \\
& \rho\left(\sigma_{4}\right)=\operatorname{diag}\left(-A^{-4}, A^{-8},-A^{-4}, A^{4},-A^{-4}, A^{4}\right)
\end{aligned}
$$


Using above mentioned $F$-matrices, we obtain

$$
\begin{aligned}
\rho\left(\sigma_{2}\right)= & \left(\begin{array}{cccccc}
\frac{A^{8}}{\Delta_{2}} & 0 & -\frac{A^{4}}{\sqrt{\Delta_{2}}} & 0 & \frac{\sqrt{\Delta_{4}}}{A^{4} \Delta_{2}} & 0 \\
0 & -A^{-4} & 0 & 0 & 0 & 0 \\
-\frac{A^{4}}{\sqrt{\Delta_{2}}} & 0 & \frac{\Delta_{5}}{\Delta_{2} \Delta_{3}} & 0 & \frac{d}{A^{8} \Delta_{3}} \sqrt{\frac{\Delta_{4}}{\Delta_{2}}} & 0 \\
0 & 0 & 0 & A^{8} \frac{d}{\Delta_{3}} & 0 & \frac{\sqrt{d \Delta_{5}}}{\Delta_{3}} \\
\frac{\sqrt{\Delta_{4}}}{A^{4} \Delta_{2}} & 0 & \frac{d}{A^{8} \Delta_{3}} \sqrt{\frac{\Delta_{4}}{\Delta_{2}}} & 0 & \frac{d}{A^{16} \Delta_{2} \Delta_{3}} & 0 \\
0 & 0 & 0 & \frac{\sqrt{d \Delta_{5}}}{\Delta_{3}} & 0 & -\frac{d}{A^{8} \Delta_{3}}
\end{array}\right) \\
\rho\left(\sigma_{3}\right)= & \left(\begin{array}{cccccc}
-A^{-4} & 0 & 0 & 0 & 0 & 0 \\
0 & \frac{A^{8}}{\Delta_{2}} & -\frac{A^{4}}{\sqrt{\Delta_{2}}} & \frac{\sqrt{\Delta_{4}}}{A^{4} \Delta_{2}} & 0 & 0 \\
0 & -\frac{A^{4}}{\sqrt{\Delta_{2}}} & \frac{\Delta_{5}}{\Delta_{2} \Delta_{3}} & \frac{d}{A^{8} \Delta_{3}} \sqrt{\frac{\Delta_{4}}{\Delta_{2}}} & 0 & 0 \\
0 & \frac{\sqrt{\Delta_{4}}}{A^{4} \Delta_{2}} & \frac{d}{A^{8} \Delta_{3}} \sqrt{\frac{\Delta_{4}}{\Delta_{2}}} & \frac{d}{A^{16} \Delta_{2} \Delta_{3}} & 0 & 0 \\
0 & 0 & 0 & 0 & A^{8} \frac{d}{\Delta_{3}} & \frac{\sqrt{d \Delta_{5}}}{\Delta_{3}} \\
0 & 0 & 0 & 0 & \frac{\sqrt{d \Delta_{5}}}{\Delta_{3}} & -\frac{d}{A^{8} \Delta_{3}}
\end{array}\right)
\end{aligned}
$$

We can see that these satisfies (4.2), and

$$
\rho\left(\sigma_{1} \sigma_{2} \sigma_{3} \sigma_{4} \sigma_{4} \sigma_{3} \sigma_{2} \sigma_{1}\right)=A^{-16} \cdot \mathbf{1}
$$

The twist is represented as (5.7).

\subsection{Topological Entanglement Entropy of Spin-1 Quasi-Particle States}

\section{龙 2-Quasi-Particle State}

We start from the 2-quasi-particle state $\left|\mathrm{qp}_{2}\right\rangle$ (5.1). Here we suppose that the first quasi-particle belongs to Alice and the second to Bob; $A=\{1\}$ and $B=\{2\}$. By use of (3.12) the reduced density matrix is depicted as

$$
\rho_{\{1\}}=\frac{1}{d^{2}-1} \stackrel{2}{ }
$$

from which (3.13) gives

$$
S_{\{1\}}^{\text {topo }}=S_{\{1\}}=\log \left(d^{2}-1\right)
$$

Recalling the topological entanglement entropy (4.19) of the two spin- $1 / 2$ quasi-particle states, we see that $d$ is replaced with $d^{2}-1$ which is nothing but the quantum dimension of the spin-1quasi-particle who intertwines Alice and Bob. 
度 3-Quasi-Particle State

In the 3-quasi-particle state $\left|\mathrm{qp}_{3}\right\rangle$ (5.2), we suppose that the owner of the first and the second quasi-particles is Alice while the third is Bob; $A=\{1,2\}$ and $B=\{3\}$. Taking a trace, i.e., connecting both ends of the Wilson line of the third particle 3.12 , we get

$$
\rho_{\{1,2\}}=\frac{d}{\left(d^{2}-1\right)\left(d^{2}-2\right)} \overbrace{2}^{2}
$$

By definition (3.13), we get the topological entanglement entropy

$$
S_{\{1,2\}}^{\text {topo }}=S_{\{1,2\}}=\log \left(d^{2}-1\right)
$$

This agrees with the logarithm of the quantum dimension of the spin-1 quasi-particle which intertwines Alice's and Bob's quasi-particles.

\section{南 4-Quasi-Particle State}

We compute the entanglement entropy for the 4-quasi-particle states defined by

$$
|\Psi\rangle=p_{0}|0\rangle+p_{1}|1\rangle+p_{2}|2\rangle
$$

Here bases $|a\rangle$ are defined in (5.3), and $\left|p_{0}\right|^{2}+\left|p_{1}\right|^{2}+\left|p_{2}\right|^{2}=1$. As the first example, we group four quasi-particles as $A=\{1,2\}$ and $B=\{3,4\}$. Through (3.12) we get the Alice's reduced density matrix as

$$
\rho_{\{1,2\}}=\frac{\left|p_{0}\right|^{2}}{d^{2}-1} \overbrace{2}+\frac{\left|p_{1}\right|^{2} d}{\left(d^{2}-1\right)\left(d^{2}-2\right)} \bigcap_{2}^{2}+\frac{\left|p_{2}\right|^{2}}{d^{4}-3 d^{2}+1} \overbrace{2}^{2}
$$

The entanglement entropy (3.13) can be computed as

$$
S_{\{1,2\}}=-\left|p_{0}\right|^{2} \log \left|p_{0}\right|^{2}-\left|p_{1}\right|^{2} \log \left(\frac{\left|p_{1}\right|^{2}}{d^{2}-1}\right)-\left|p_{2}\right|^{2} \log \left(\frac{\left|p_{2}\right|^{2}}{d^{4}-3 d^{2}+1}\right)
$$

As bases (5.3) have a form of (3.2) due to (7.8), the topological entanglement entropy (3.9) is given by

$$
S_{\{1,2\}}^{\text {topo }}=\left|p_{1}\right|^{2} \log \left(d^{2}-1\right)+\left|p_{2}\right|^{2} \log \left(d^{4}-3 d^{2}+1\right)
$$

We see the appearance of quantum dimension $d_{2}=d^{2}-1$ and $d_{4}=d^{4}-3 d^{2}+1$ of quasi-particles which respectively intertwines Alice and Bob in the state $|1\rangle$ and $|2\rangle$. 
When we assume that $A=\{2,3\}$ and $B=\{1,4\}$, it is convenient to change bases using the $F$-matrix (5.4). We have another expression of (5.17);

$$
\begin{aligned}
& \left.\left.|\Psi\rangle=\tilde{p_{0}} \frac{1}{d^{2}-1} \bigcup^{2}\right)+\widetilde{p_{1}} \frac{d}{\left(d^{2}-2\right) \sqrt{d^{2}-1}} \bigcup_{2}^{2}\right)^{2} \\
& +\widetilde{p_{2}} \frac{1}{\sqrt{d^{4}-3 d^{2}+1}} \underbrace{2}_{4})^{2}
\end{aligned}
$$

where

$$
\begin{aligned}
& \widetilde{p_{0}}=p_{0} \frac{1}{d^{2}-1}+p_{1} \frac{1}{\sqrt{d^{2}-1}}+p_{2} \frac{\sqrt{d^{4}-3 d^{2}+1}}{d^{2}-1} \\
& \widetilde{p_{1}}=p_{0} \frac{1}{\sqrt{d^{2}-1}}+p_{1} \frac{d^{2}-3}{d^{2}-2}-p_{2} \frac{1}{d^{2}-2} \sqrt{\frac{d^{4}-3 d^{2}+1}{d^{2}-1}} \\
& \widetilde{p_{2}}=p_{0} \frac{\sqrt{d^{4}-3 d^{2}+1}}{d^{2}-1}-p_{1} \frac{1}{d^{2}-2} \sqrt{\frac{d^{4}-3 d^{2}+1}{d^{2}-1}}+p_{2} \frac{1}{\left(d^{2}-1\right)\left(d^{2}-2\right)}
\end{aligned}
$$

See that $\left|\widetilde{p_{0}}\right|^{2}+\left|\widetilde{p_{1}}\right|^{2}+\left|\widetilde{p_{2}}\right|^{2}=1$. By the same computation, we obtain the (topological) entanglement entropy as follows;

$$
\begin{gathered}
S_{\{2,3\}}=-\left|\widetilde{p_{0}}\right|^{2} \log \left|\widetilde{p_{0}}\right|^{2}-\left|\widetilde{p_{1}}\right|^{2} \log \left(\frac{\left|\widetilde{p_{1}}\right|^{2}}{d^{2}-1}\right)-\left|\widetilde{p_{2}}\right|^{2} \log \left(\frac{\left|\widetilde{p_{2}}\right|^{2}}{d^{4}-3 d^{2}+1}\right) \\
S_{\{2,3\}}^{\text {topo }}=\left|\widetilde{p_{1}}\right|^{2} \log \left(d^{2}-1\right)+\left|\widetilde{p_{2}}\right|^{2} \log \left(d^{4}-3 d^{2}+1\right)
\end{gathered}
$$

which is consistent with our interpretation of the topological entanglement entropy; when we look at (5.21), we find that parameters $\widetilde{p_{1}}$ and $\widetilde{p_{2}}$ respectively denote probabilities that the spin- 1 and spin-2 quasi-particle intertwines Alice and Bob.

\subsection{Fibonacci Anyon}

The spin-1 quasi-particle in the $S U(2)_{3}$ theory is known as the Fibonacci anyon [49]. This is because the dimension of the Hilbert space of $n$-quasi-particle with spin-1, i.e., the number of paths from $(0,0)$ to $(n, 0)$ in Fig. 2, coincides with the Fibonacci number. For instance, among three states of 4-quasi-particles (5.3), the state $|2\rangle$ is not admissible (2.24); the norm of the diagram for $|2\rangle$ vanishes, and it is a null state. Setting $A=\mathrm{ie}^{\pi \mathrm{i} / 10}$ and $d$ to be the golden-ratio $d=$ $2 \cos (\pi / 5)=\frac{1+\sqrt{5}}{2} \equiv \tau$ in $(5.6)$ we obtain 2-dimensional representation of the braid operators on 
$\{|0\rangle,|1\rangle\}$ as

$$
\begin{gathered}
\rho\left(\sigma_{1}\right)=\rho\left(\sigma_{3}\right)=\left(\begin{array}{cc}
\mathrm{e}^{-\frac{4}{5} \pi \mathrm{i}} & 0 \\
0 & \mathrm{e}^{\frac{3}{5} \pi \mathrm{i}}
\end{array}\right) \\
\rho\left(\sigma_{2}\right)=\left(\begin{array}{cc}
\frac{\mathrm{e}^{\frac{4}{5} \pi \mathrm{i}}}{\tau} & -\frac{\mathrm{e}^{\frac{2}{5} \pi \mathrm{i}}}{\sqrt{\tau}} \\
-\frac{\mathrm{e}^{\frac{2}{5} \pi \mathrm{i}}}{\sqrt{\tau}} & -\frac{1}{\tau}
\end{array}\right) \\
\rho(\theta)=\mathrm{e}^{\frac{4}{5} \pi \mathrm{i}}
\end{gathered}
$$

which are given in Ref. 49 assuming the unitarity condition.

A dimension of the Hilbert space of 5-quasi-particle states becomes 3; the norms of diagrams in the states $|12\rangle,|21\rangle$, and $|22\rangle$ vanish due to $\Delta_{4}=0$, and these states are not admissible (2.24). Note that only $|22\rangle$ is forbidden for a case of $K=4$. Then on bases $\{|01\rangle,|10\rangle,|11\rangle\}$, the braiding matrices (5.11) and (5.12) reduce to

$$
\begin{aligned}
& \rho\left(\sigma_{1}\right)=\operatorname{diag}\left(\mathrm{e}^{-\frac{4}{5} \pi \mathrm{i}}, \mathrm{e}^{\frac{3}{5} \pi \mathrm{i}}, \mathrm{e}^{\frac{3}{5} \pi \mathrm{i}}\right) \\
& \rho\left(\sigma_{2}\right)=\left(\begin{array}{ccc}
\frac{\mathrm{e}^{\frac{4}{5} \pi \mathrm{i}}}{\tau} & 0 & -\frac{\mathrm{e}^{\frac{2}{5} \pi \mathrm{i}}}{\sqrt{\tau}} \\
0 & \mathrm{e}^{\frac{3}{5} \pi \mathrm{i}} & 0 \\
-\frac{\mathrm{e}^{\frac{2}{5} \pi \mathrm{i}}}{\sqrt{\tau}} & 0 & -\frac{1}{\tau}
\end{array}\right) \\
& \rho\left(\sigma_{3}\right)=\left(\begin{array}{ccc}
\mathrm{e}^{\frac{3}{5} \pi \mathrm{i}} & 0 & 0 \\
0 & \frac{\mathrm{e}^{\frac{4}{5} \pi \mathrm{i}}}{\tau} & -\frac{\mathrm{e}^{\frac{2}{5} \pi \mathrm{i}}}{\sqrt{\tau}} \\
0 & -\frac{\mathrm{e}^{\frac{2}{5} \pi \mathrm{i}}}{\sqrt{\tau}} & -\frac{1}{\tau}
\end{array}\right) \\
& \rho\left(\sigma_{4}\right)=\operatorname{diag}\left(\mathrm{e}^{\frac{3}{5} \pi \mathrm{i}}, \mathrm{e}^{-\frac{4}{5} \pi \mathrm{i}}, \mathrm{e}^{\frac{3}{5} \pi \mathrm{i}}\right)
\end{aligned}
$$

On the topological entanglement entropy (3.9), we recall that the quantum dimension of quasiparticles with spin- $1 / 2,1$, and $3 / 2$ are respectively given by $d_{1}=\tau, d_{2}=\tau$, and $d_{3}=1$. Thus the topological entanglement entropy always takes a form of $\left|p_{\tau}\right|^{2} \log \tau$ where $\left|p_{\tau}\right|^{2}$, satisfying $0 \leq\left|p_{\tau}\right|^{2} \leq 1$, is a probability that Alice and Bob are intertwined through quasi-particle with spin- $1 / 2$ or spin- 1 .

\section{QUBIT FROM Different SPECIES OF QUASI-PARTICleS}

In previous sections, we have only considered states which are constructed from quasi-particles with same spin; spin-1/2 and spin-1. In this section we shall propose another candidate of topological qubit which is composed from 4-quasi-particles with different spins. Namely we set 
$1<n \leq K-1$, and study states constituted from two spin-1/2 quasi-particles and two spin$n / 2$ quasi-particles. We set bases as follows;

$$
\begin{aligned}
& \left|0_{1}\right\rangle=\frac{\sqrt{\Delta_{n-1}}}{\Delta_{n}} \bigcup_{n-1}^{n} y^{n} \\
& \left|1_{1}\right\rangle=\frac{1}{\sqrt{\Delta_{n+1}}} \bigcup_{n+1}^{n} \bigcup^{n} \\
& \left|0_{2}\right\rangle=\frac{\sqrt{\Delta_{n-1}}}{\Delta_{n}} \bigcup_{n-1}^{1} \bigcup^{n} \\
& \left|1_{2}\right\rangle=\frac{1}{\sqrt{\Delta_{n+1}}} \bigcup_{n+1}^{1} y^{n} \\
& \left|0_{3}\right\rangle=\frac{\sqrt{\Delta_{n-1}}}{\Delta_{n}} \bigcup_{n-1}^{n} \bigcup^{n} \\
& \left|1_{3}\right\rangle=\frac{1}{\sqrt{\Delta_{n+1}}} \bigcup_{n+1}^{n} \bigcup^{1} \\
& \left|0_{4}\right\rangle=\frac{\sqrt{\Delta_{n-1}}}{\Delta_{n}} \bigcup_{n-1}^{n} Y^{1} \\
& \left|1_{4}\right\rangle=\frac{1}{\sqrt{\Delta_{n+1}}} \bigcup_{n+1}^{n} Y^{1} \\
& \left|0_{5}\right\rangle=\frac{1}{\sqrt{d \Delta_{n}}} \bigcup_{1} \bigcup_{n} \\
& \left|1_{5}\right\rangle=\sqrt{\frac{d \Delta_{n-1}}{\Delta_{n} \Delta_{n+1}}} \bigcup_{2}^{1} \mho^{n} \\
& \left|0_{6}\right\rangle=\frac{1}{\sqrt{d \Delta_{n}}} \bigcup_{n} \bigcup_{1} \\
& \left|1_{6}\right\rangle=\sqrt{\frac{d \Delta_{n-1}}{\Delta_{n} \Delta_{n+1}}} \bigcup_{2}^{n} y^{1}
\end{aligned}
$$

Dual states $\left\langle i_{a}\right|$ are upside down of $\left|i_{a}\right\rangle$ with the same normalization factor. A condition (2.21) proves that these states are physical states, i.e., norm of each diagram is positive. Normalizations are chosen so that

$$
\left\langle i_{a} \mid j_{a}\right\rangle=\delta_{i, j}
$$

for $i, j \in\{0,1\}$ and $1 \leq a \leq 6$.

To compute explicit representation of braid operators, we prepare the $F$-matrices. Due to the definition of the idempotent, we find

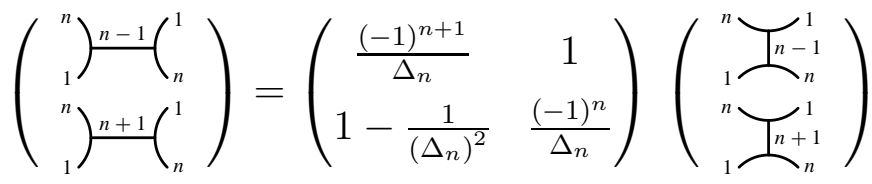

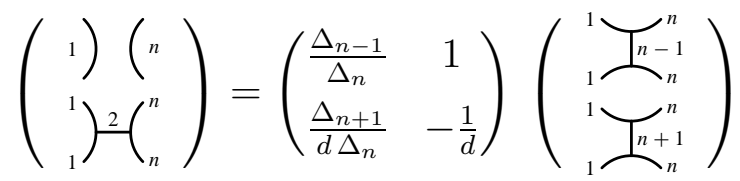


Using these $F$-matrices, we can compute the actions of braid operators on $\left\{\left|0_{1}\right\rangle,\left|1_{1}\right\rangle,\left|0_{2}\right\rangle,\left|1_{2}\right\rangle, \cdots\left|0_{6}\right\rangle,\left|1_{6}\right\rangle\right\}$ are computed. The actions of $\sigma_{1}$ and $\sigma_{3}$ follow from (2.26) as

$$
\begin{aligned}
\rho\left(\sigma_{1}\right) & =\left(\begin{array}{cccccc}
0 & \mathbf{R}_{1} & 0 & 0 & 0 & 0 \\
\mathbf{R}_{1} & 0 & 0 & 0 & 0 & 0 \\
0 & 0 & 0 & \mathbf{R}_{1} & 0 & 0 \\
0 & 0 & \mathbf{R}_{1} & 0 & 0 & 0 \\
0 & 0 & 0 & 0 & \mathbf{R}_{2} & 0 \\
0 & 0 & 0 & 0 & 0 & \mathbf{R}_{3}
\end{array}\right) \\
\rho\left(\sigma_{3}\right) & =\left(\begin{array}{cccccc}
0 & 0 & \mathbf{R}_{1} & 0 & 0 & 0 \\
0 & 0 & 0 & \mathbf{R}_{1} & 0 & 0 \\
\mathbf{R}_{1} & 0 & 0 & 0 & 0 & 0 \\
0 & \mathbf{R}_{1} & 0 & 0 & 0 & 0 \\
0 & 0 & 0 & 0 & \mathbf{R}_{3} & 0 \\
0 & 0 & 0 & 0 & 0 & \mathbf{R}_{2}
\end{array}\right)
\end{aligned}
$$

where 0 denotes a 2 by 2 null matrix, and $R_{a}$ are 2 by 2 diagonal matrices defined by

$$
\begin{gathered}
\mathbf{R}_{1}=\operatorname{diag}\left(-A^{-n-2}, A^{n}\right) \\
\mathbf{R}_{2}=\operatorname{diag}\left(-A^{-3}, A\right) \\
\mathbf{R}_{3}=\operatorname{diag}\left((-1)^{n} A^{-n^{2}-2 n},(-1)^{n+1} A^{-n^{2}-2 n+4}\right)
\end{gathered}
$$

Then by use of above $F$-matrices we obtain the representations of $\sigma_{2}$ as

$$
\rho\left(\sigma_{2}\right)=\left(\begin{array}{cccccc}
0 & 0 & 0 & 0 & \mathbf{B}_{1} & 0 \\
0 & \mathbf{B}_{2} & 0 & 0 & 0 & 0 \\
0 & 0 & \mathbf{B}_{3} & 0 & 0 & 0 \\
0 & 0 & 0 & 0 & 0 & \mathbf{B}_{1} \\
\mathbf{B}_{1}^{\mathrm{T}} & 0 & 0 & 0 & 0 & 0 \\
0 & 0 & 0 & \mathbf{B}_{1}^{\mathrm{T}} & 0 & 0
\end{array}\right)
$$

where 0 is a 2 by 2 null matrix as before, and $\mathbf{B}_{a}$ are

$$
\begin{gathered}
\mathbf{B}_{1}=\frac{1}{\sqrt{d \Delta_{n}}}\left(\begin{array}{cc}
-A^{n+2} \sqrt{\Delta_{n-1}} & A^{n-2} \sqrt{\Delta_{n+1}} \\
A^{-n} \sqrt{\Delta_{n+1}} & A^{-n-4} \sqrt{\Delta_{n-1}}
\end{array}\right) \\
\mathbf{B}_{2}=\frac{1}{A \Delta_{n}}\left(\begin{array}{cc}
A^{2} \Delta_{n}+\Delta_{n-1} & \sqrt{\Delta_{n+1} \Delta_{n-1}} \\
\sqrt{\Delta_{n+1} \Delta_{n-1}} & -\left(A^{-2} \Delta_{n}+\Delta_{n-1}\right)
\end{array}\right) \\
\mathbf{B}_{3}=(-1)^{n+1} A^{-n^{2}-2 n+3} \mathbf{B}_{2}
\end{gathered}
$$

We mean that $\mathbf{B}_{1}^{\mathrm{T}}$ is a transpose of $\mathbf{B}_{1}$.

We note that $\rho\left(\sigma_{a}^{2}\right)$ for all $a$ are block diagonal

$$
\begin{gathered}
\rho\left(\sigma_{1}^{2}\right)=\operatorname{diag}\left(\mathbf{R}_{1}^{2}, \mathbf{R}_{1}^{2}, \mathbf{R}_{1}^{2}, \mathbf{R}_{1}^{2}, \mathbf{R}_{2}^{2}, \mathbf{R}_{3}^{2}\right) \\
\rho\left(\sigma_{2}^{2}\right)=\operatorname{diag}\left(\mathbf{B}_{1} \mathbf{B}_{1}^{\mathrm{T}}, \mathbf{B}_{2}^{2}, \mathbf{B}_{3}^{2}, \mathbf{B}_{1} \mathbf{B}_{1}^{\mathrm{T}}, \mathbf{B}_{1}^{\mathrm{T}} \mathbf{B}_{1}, \mathbf{B}_{1}^{\mathrm{T}} \mathbf{B}_{1}\right) \\
\rho\left(\sigma_{3}^{2}\right)=\operatorname{diag}\left(\mathbf{R}_{1}^{2}, \mathbf{R}_{1}^{2}, \mathbf{R}_{1}^{2}, \mathbf{R}_{1}^{2}, \mathbf{R}_{3}^{2}, \mathbf{R}_{2}^{2}\right)
\end{gathered}
$$


This fact shows that, when we restrict braiding operators generated from even powers of braid operators $\left\{\sigma_{1}^{2}, \sigma_{2}^{2}, \sigma_{3}^{2}\right\}$, all the 2-dimensional spaces $\left\{\left|0_{a}\right\rangle,\left|1_{a}\right\rangle\right\}$ are preserved. Then these 2dimensional space may be treated as qubit, and block matrices in (6.6) are treated as unitary operators on these spaces.

It should be noted that other sets of braid operators preserves 2-dimensional space, e.g.

- A set of $\left\{\sigma_{1}^{2}, \sigma_{2}, \sigma_{3}^{2}\right\}$ preserves $\left|i_{2}\right\rangle$ and $\left|i_{3}\right\rangle$.

- A set of $\left\{\sigma_{1}, \sigma_{2}^{2}, \sigma_{3}\right\}$ preserves $\left|i_{5}\right\rangle$ and $\left|i_{6}\right\rangle$.

It is remarked that some of the braiding matrices are given in Ref. 2 based on explicit form of the correlation functions.

\section{Many-Quasi-Particle States and Quantum $6 j$ Symbol}

Up to now, we have derived braiding matrices for systems of small numbers of quasi-particles based on the skein theory. We have derived all $F$-matrices in an elementary and combinatorial way based on the Jones-Wenzl idempotent. Although, explicit forms of the general $F$-matrices are known as the quantum $6 j$ system, and we can construct the braiding matrices for arbitrary numbers of quasi-particles.

\subsection{Quantum $6 j$ Symbol}

We borrow results on the quantum $6 j$ symbol from Ref. 31 (see also Refs. 7, 42, 43). We define $q$-integer and $q$-factorial by

$$
\begin{gathered}
{[n]=\frac{A^{2 n}-A^{-2 n}}{A^{2}-A^{-2}}=(-1)^{n-1} \Delta_{n-1}} \\
{[n] !=\prod_{i=1}^{n}[i]}
\end{gathered}
$$

The quantum $6 j$-symbol comes from the following Temperley-Lieb recoupling diagram;

$$
)_{a}^{b}(_{d}^{c}=\sum_{i}\left\{\begin{array}{lll}
a & b & i \\
c & d & j
\end{array}\right\} \overbrace{a}^{b} \overbrace{d}^{c}
$$

We have a complicated formula for the quantum $6 j$-symbol, which was first computed in Ref. 33;

$$
\left\{\begin{array}{lll}
a & b & i \\
c & d & j
\end{array}\right\}=\frac{\Delta_{i}}{\theta(a, d, i) \theta(b, c, i)} \text { Tet }\left[\begin{array}{lll}
a & b & i \\
c & d & j
\end{array}\right]
$$

Here $\theta(a, b, c)$ is the $\theta$-net

$$
\theta(a, b, c)=\bigodot_{c}^{a}
$$


which is given explicitly as

$$
\theta(a, b, c)=(-1)^{i+j+k} \frac{[i+j+k+1] ![i] ![j] ![k] !}{[i+j] ![j+k] ![i+k] !}
$$

with

$$
i=\frac{-a+b+c}{2} \quad j=\frac{a-b+c}{2} \quad k=\frac{a+b-c}{2}
$$

The tetrahedral net is defined by

$$
\operatorname{Tet}\left[\begin{array}{ccc}
A & B & E \\
C & D & F
\end{array}\right]={ }_{D}
$$

which is known to be

$\operatorname{Tet}\left[\begin{array}{ccc}A & B & E \\ C & D & F\end{array}\right]=\frac{\prod_{i, j}\left[b_{j}-a_{i}\right] !}{[A] ![B] ![C] ![D] ![E] ![F] !}$

$$
\times \sum_{\max \left\{a_{i}\right\} \leq s \leq \min \left\{b_{j}\right\}} \frac{(-1)^{s}[s+1] !}{\prod_{i}\left[s-a_{i}\right] ! \prod_{j}\left[b_{j}-s\right] !}
$$

with

$$
\begin{aligned}
a_{1} & =\frac{1}{2}(A+D+E) & b_{1} & =\frac{1}{2}(B+D+E+F) \\
a_{2} & =\frac{1}{2}(B+C+E) & b_{2} & =\frac{1}{2}(A+C+E+F) \\
a_{3} & =\frac{1}{2}(A+B+F) & b_{3} & =\frac{1}{2}(A+B+C+D) \\
a_{4} & =\frac{1}{2}(C+D+F) & &
\end{aligned}
$$

As a simple application of the quantum $6 j$-symbol (7.1), we have

$$
\left.\left.\frac{a}{b}=\sum_{c} \frac{\Delta_{c}}{\theta(a, b, c)}\right)_{b}^{a}\right){ }_{b}^{a}
$$

Note that the $\theta$-net appears from

$$
\bigcap_{d}^{a} c=\delta_{a, d} \frac{\theta(a, b, c)}{\Delta_{a}} a
$$

The quantum $6 j$ symbol has symmetries;

$$
\left\{\begin{array}{lll}
a & b & i \\
c & d & j
\end{array}\right\}=\left\{\begin{array}{lll}
c & d & i \\
a & b & j
\end{array}\right\}
$$


which follows from a $180^{\circ}$ rotation of (7.1). Also we have

$$
\begin{aligned}
\frac{\theta(a, d, i)}{\Delta_{i}}\left\{\begin{array}{lll}
a & b & i \\
c & d & j
\end{array}\right\} & =\frac{\theta(a, b, j)}{\Delta_{b}}\left\{\begin{array}{lll}
j & c & b \\
i & a & d
\end{array}\right\} \\
& =\frac{\theta(c, d, j)}{\Delta_{c}}\left\{\begin{array}{lll}
d & i & c \\
b & j & a
\end{array}\right\}
\end{aligned}
$$

which comes from applications of the $6 j$-symbol to the following net;
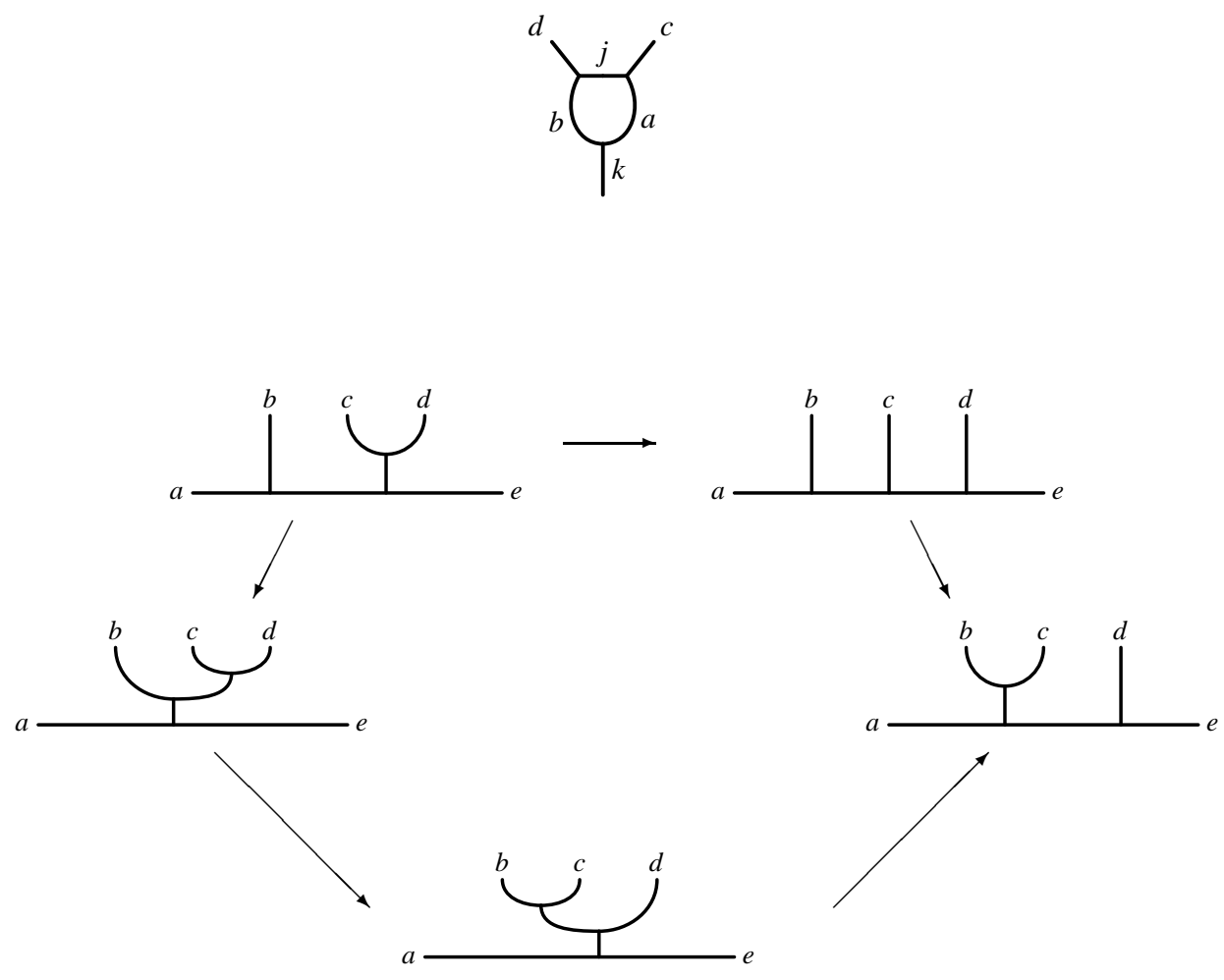

Figure 3: Pentagon identity

Recursive use of the recoupling diagram (7.1) gives us the orthogonality relation;

$$
\sum_{i}\left\{\begin{array}{lll}
a & b & i \\
c & d & j
\end{array}\right\}\left\{\begin{array}{lll}
d & a & k \\
b & c & i
\end{array}\right\}=\delta_{j, k}
$$

Well known is the pentagon equation, or the Biedenharn-Elliott identity, which follows from Fig. (3);

$$
\sum_{m}\left\{\begin{array}{llc}
a & i & m \\
d & e & j
\end{array}\right\}\left\{\begin{array}{ccc}
b & c & \ell \\
d & m & i
\end{array}\right\}\left\{\begin{array}{ccc}
b & \ell & k \\
e & a & m
\end{array}\right\}=\left\{\begin{array}{lll}
b & c & k \\
j & a & i
\end{array}\right\}\left\{\begin{array}{lll}
k & c & \ell \\
d & e & j
\end{array}\right\}
$$

Amongst others useful identity we use [7]

$$
\left(\lambda_{a}^{d i}\right)^{-1} \lambda_{j}^{c d}\left\{\begin{array}{lll}
a & b & i \\
c & d & j
\end{array}\right\}=\sum_{k}\left\{\begin{array}{lll}
a & b & k \\
d & c & j
\end{array}\right\}\left(\lambda_{k}^{b d}\right)^{-1}\left\{\begin{array}{lll}
d & b & i \\
c & a & k
\end{array}\right\}
$$


which is the $B$-matrix [45]. To prove this, we first compute as

$$
\rangle_{a}^{b}\left\langle\left\langle_{c}^{d}=\lambda_{j}^{c d}\right\rangle_{a}^{b} q_{c}^{d}=\sum_{i} \lambda_{j}^{c d}\left\{\begin{array}{lll}
a & b & i \\
c & d & j
\end{array}\right\}{ }^{b}\right\rangle_{c}^{d}
$$

On the other hand, the left hand side is transformed as

$$
\begin{aligned}
& \rangle_{a}^{b}\langle_{c}^{d}=\sum_{k}\left\{\begin{array}{lll}
a & b & k \\
d & c & j
\end{array}\right\} \underbrace{b}_{a} \nearrow_{c}^{d}=\sum_{k}\left\{\begin{array}{lll}
a & b & k \\
d & c & j
\end{array}\right\}\left(\lambda_{k}^{b d}\right)^{-1} \overbrace{c}^{b} \\
& =\sum_{i, k}\left\{\begin{array}{lll}
a & b & k \\
d & c & j
\end{array}\right\}\left(\lambda_{k}^{b d}\right)^{-1}\left\{\begin{array}{lll}
c & a & i \\
d & b & k
\end{array}\right\} \quad{ }_{a}^{b}{\overline{R_{i}}}_{c}^{d} \\
& =\sum_{i, k}\left\{\begin{array}{lll}
a & b & k \\
d & c & j
\end{array}\right\}\left(\lambda_{k}^{b d}\right)^{-1}\left\{\begin{array}{lll}
d & b & i \\
c & a & k
\end{array}\right\} \quad{ }_{a}^{b} \vec{F}_{c}^{d} \\
& =\left.\sum_{i, k}\left\{\begin{array}{lll}
a & b & k \\
d & c & j
\end{array}\right\}\left(\lambda_{k}^{b d}\right)^{-1}\left\{\begin{array}{lll}
d & b & i \\
c & a & k
\end{array}\right\} \lambda_{a}^{d i}{ }_{a}^{b}\right|_{c} ^{d}
\end{aligned}
$$

Combining these expressions, we obtain (7.13).

\subsection{Braid Relations for 4-Quasi-Particles with Arbitrary Spins}

We generalize results in previous sections. We define the state

$$
\left|i_{a b ; c d}\right\rangle=\sqrt{\frac{\Delta_{i}}{\theta(a, b, i) \theta(c, d, i)}} \bigcup_{i}^{b} \bigcup^{c}
$$

The dimension of the Hilbert space, i.e., the number of $i$ for fixed $\{a, b, c, d\}$, is the number of $i$ 's such that vertices $(a, b, i)$ and $(c, d, i)$ satisfy the admissible condition (2.24). The orthonormality follows from (7.8);

$$
\left\langle i_{a b ; c d} \mid j_{a b ; c d}\right\rangle=\delta_{i, j}
$$

The braiding matrices on the space spanned by $\left|i_{a b ; c d}\right\rangle$ can be computed by use of the quantum $6 j$-symbol (7.1) and the twist formula (2.26). For instance, we compute as follows;

$$
\begin{aligned}
\sigma_{2} \bigcup_{i}^{a} y^{d} & =\sum_{k}\left\{\begin{array}{lll}
a & b & k \\
c & d & i
\end{array}\right\} \\
& =\sum_{k}\left\{\begin{array}{lll}
a & b & k \\
c & d & i
\end{array}\right\}\left(\lambda_{k}^{b c}\right)^{-1} \underbrace{a}_{k}\}^{d} \\
& =\sum_{k}\left\{\begin{array}{lll}
a & b & k \\
c & d & i
\end{array}\right\}\left(\lambda_{k}^{b c}\right)^{-1}\left\{\begin{array}{lll}
c & b & j \\
d & a & k
\end{array}\right\} \underbrace{c}_{j} Y^{b}
\end{aligned}
$$


Applying (7.13) and using a normalization factor, we obtain $\rho\left(\sigma_{2}\right)$. In summary, we have the braiding matrices as

$$
\begin{gathered}
\rho\left(\sigma_{1}\right)\left|i_{a b ; c d}\right\rangle=\left(\lambda_{i}^{a b}\right)^{-1}\left|i_{b a ; c d}\right\rangle \\
\rho\left(\sigma_{2}\right)\left|i_{a b ; c d}\right\rangle=\sum_{j} \sqrt{\frac{\Delta_{i} \theta(a, c, j) \theta(b, d, j)}{\Delta_{j} \theta(a, b, i) \theta(c, d, i)}}\left(\lambda_{a}^{c j}\right)^{-1} \lambda_{i}^{c d}\left\{\begin{array}{lll}
a & b & j \\
d & c & i
\end{array}\right\}\left|j_{a c ; b d}\right\rangle \\
\rho\left(\sigma_{3}\right)\left|i_{a b ; c d}\right\rangle=\left(\lambda_{i}^{c d}\right)^{-1}\left|i_{a b ; d c}\right\rangle
\end{gathered}
$$

See that the quantum $6 j$-symbol with the square root term in $\rho\left(\sigma_{2}\right)$ coincides with the unitary matrix in Ref. 32. Twist operators are

$$
\rho\left(\theta_{1}\right)\left|i_{a b ; c d}\right\rangle=\lambda_{0}^{a a}\left|i_{a b ; c d}\right\rangle
$$

and so on.

We have

$$
\begin{aligned}
& \rho\left(\sigma_{1} \sigma_{2} \sigma_{3}^{2} \sigma_{2} \sigma_{1}\right)\left|i_{a b ; c d}\right\rangle=\left(\lambda_{0}^{a a}\right)^{-2}\left|i_{a b ; c d}\right\rangle \\
& \rho\left(\left(\sigma_{1} \sigma_{2} \sigma_{3}\right)^{4}\right)\left|i_{a b ; c d}\right\rangle=\left(\lambda_{0}^{a b} \lambda_{0}^{c d}\right)^{-2}\left|i_{a b ; c d}\right\rangle
\end{aligned}
$$

\subsection{Braid Relations for Many-Quasi-Particles with Spin- $c / 2$}

We study $(n+3)$-quasi-particle with spin- $c / 2$. We set

$$
\left|s_{1} s_{2} \cdots s_{n} ; c\right\rangle=\left.\left.\left.\frac{1}{\sqrt{N_{s_{1} \cdots s_{n}}^{(c)}}} \bigcup_{s_{1}}^{c}\right|_{s_{2}} ^{c}\right|_{s_{3}} ^{c}\right|_{s_{n}} ^{c} \bigcup^{c}
$$

where $s_{i} \geq 0$. The admissible condition (2.24) is read as

$$
\begin{gathered}
c \leq s_{i}+s_{i+1} \leq 2 K-c \\
s_{i}-c \leq s_{i+1} \leq s_{i}+c \\
s_{i}+s_{i+1}+c=0 \bmod 2
\end{gathered}
$$

where we mean $s_{0}=s_{n+1}=c$. Thanks to (7.8) the normalization factor is computed as

$$
N_{s_{1} \cdots s_{n}}^{(c)}=\frac{\prod_{i=1}^{n+1} \theta\left(s_{i-1}, s_{i}, c\right)}{\prod_{i=1}^{n} \Delta_{s_{i}}}
$$

and we have

$$
\left\langle s_{1}^{\prime} s_{2}^{\prime} \cdots s_{n}^{\prime} ; c \mid s_{1} s_{2} \cdots s_{n} ; c\right\rangle=\prod_{i=1}^{n} \delta_{s_{i}, s_{i}^{\prime}}
$$

Here the dual state $\left\langle s_{1} s_{2} \cdots s_{n} ; c\right|$ is defined upside down with same normalization factor. 
Actions of the braid operators $\sigma_{i}$ can be computed in essentially same method with the previous sections. Results are as follows;

$$
\begin{gathered}
\rho\left(\sigma_{1}\right)\left|s_{1} \cdots s_{n} ; c\right\rangle=\left(\lambda_{s_{1}}^{c c}\right)^{-1}\left|s_{1} \cdots s_{n} ; c\right\rangle \\
\rho\left(\sigma_{n+2}\right)\left|s_{1} \cdots s_{n} ; c\right\rangle=\left(\lambda_{s_{n}}^{c c}\right)^{-1}\left|s_{1} \cdots s_{n} ; c\right\rangle
\end{gathered}
$$

and, for $1<i<n+1$, we have

$$
\begin{aligned}
& \rho\left(\sigma_{i+1}\right)\left|s_{1} \cdots s_{n} ; c\right\rangle \\
& =\sum_{m} \sqrt{\frac{\Delta_{s_{i}} \theta\left(m, s_{i-1}, c\right) \theta\left(s_{i+1}, m, c\right)}{\Delta_{m} \theta\left(s_{i}, s_{i-1}, c\right) \theta\left(s_{i+1}, s_{i}, c\right)}}\left(\lambda_{s_{i-1}}^{c m}\right)^{-1} \lambda_{s_{i}}^{s_{i+1} c}\left\{\begin{array}{lll}
s_{i-1} & c & m \\
s_{i+1} & c & s_{i}
\end{array}\right\} \\
& \times\left|s_{1} \cdots m \cdots s_{n} ; c\right\rangle
\end{aligned}
$$

We see that

$$
\begin{gathered}
\rho\left(\sigma_{1} \sigma_{2} \cdots \sigma_{n+1} \sigma_{n+2}^{2} \sigma_{n+1} \cdots \sigma_{2} \sigma_{1}\right)\left|s_{1} \cdots s_{n} ; c\right\rangle=\left(\lambda_{0}^{c c}\right)^{-2}\left|s_{1} \cdots s_{n} ; c\right\rangle \\
\rho\left(\left(\sigma_{1} \sigma_{2} \cdots \sigma_{n+2}\right)^{n+3}\right)\left|s_{1} \cdots s_{n} ; c\right\rangle=\left(\lambda_{0}^{c 0}\right)^{-2(n+3)}\left|s_{1} \cdots s_{n} ; c\right\rangle
\end{gathered}
$$

\section{Spin-1/2 Quasi-Particle States}

In a case of $c=1$, the admissible condition (7.20) is

$$
\begin{aligned}
& \quad 1 \leq s_{i}+s_{i+1} \leq 2 K-1 \\
& s_{i+1}=s_{i}-1 \quad \text { or } \quad s_{i+1}=s_{i}+1
\end{aligned}
$$

where we mean $s_{0}=s_{n+1}=1$. Normalization factor is written with the $\theta$-net,

$$
\theta(a, b, 1)=\Delta_{\max }(a, b)
$$

Note that the notation $\left|s_{1} s_{2} s_{3} ; 1\right\rangle$ is different from (4.12) used in 6-quasi-particle case; $|010 ; 1\rangle$, $|012 ; 1\rangle$, and $|210 ; 1\rangle$ respectively coincide with $|00\rangle,|01\rangle$, and $|10\rangle$ in (4.12). Remaining two states, $|212 ; 1\rangle$ and $|232 ; 1\rangle$, are related to $|11\rangle$ and $|C\rangle$ in (4.12) through the $F$-matrix (4.13).

The braid matrices follows from (7.23) $-(7.25)$, and non-zero quantum $6 j$-symbols therein are

$$
\begin{aligned}
\left\{\begin{array}{ccc}
a & 1 & a+1 \\
a+2 & 1 & a+1
\end{array}\right\} & =\left\{\begin{array}{lll}
a & 1 & a+1 \\
a & 1 & a-1
\end{array}\right\}=\left\{\begin{array}{ccc}
a & 1 & a-1 \\
a-2 & 1 & a-1
\end{array}\right\}=1 \\
\left\{\begin{array}{lll}
a & 1 & a-1 \\
a & 1 & a+1
\end{array}\right\} & =\frac{\Delta_{a-1} \Delta_{a+1}}{\left(\Delta_{a}\right)^{2}} \\
\left\{\begin{array}{lll}
a & 1 & a \pm 1 \\
a & 1 & a \pm 1
\end{array}\right\} & =\mp \frac{1}{\Delta_{a}}
\end{aligned}
$$




\section{应 Spin-1 Quasi-Particle States}

The admissible condition (7.20) with $c=2$ is read as

$$
\begin{gathered}
2 \leq s_{i}+s_{i+1} \leq 2 K-2 \\
s_{i+1} \in\left\{s_{i} \pm 2, s_{i}\right\}
\end{gathered}
$$

and $s_{0}=s_{n+1}=2$. The normalization factor is calculated by use of the following $\theta$-net;

$$
\theta(2 a, 2 b, 2)= \begin{cases}\frac{\Delta_{2 a} \Delta_{2 a+1}}{d \Delta_{2 a-1}} & \text { for } a=b \\ \Delta_{2 \max (a, b)} & \text { for }|a-b|=1 \\ 0 & \text { others }\end{cases}
$$

States, $\left|\frac{s_{1}}{2}\right\rangle(5.3)$, and $\left|\frac{s_{1}}{2} \frac{s_{2}}{2}\right\rangle(5.8)$, respectively correspond to $\left|s_{1} ; 2\right\rangle$, and $\left|s_{1} s_{2} ; 2\right\rangle$. Non-zero $6 j$-symbols are;

$$
\begin{aligned}
& \left\{\begin{array}{ccc}
2 a & 2 & 2 a \pm 2 \\
2 a \pm 4 & 2 & 2 a \pm 2
\end{array}\right\}=\left\{\begin{array}{ccc}
2 a & 2 & 2 a+2 \\
2 a+2 & 2 & 2 a
\end{array}\right\}=1 \\
& \left\{\begin{array}{ccc}
2 a & 2 & 2 a+2 \\
2 a+2 & 2 & 2 a+2
\end{array}\right\}=\frac{d}{\Delta_{2 a+1}} \quad\left\{\begin{array}{ccc}
2 a & 2 & 2 a \\
2 a+2 & 2 & 2 a+2
\end{array}\right\}=\frac{\Delta_{2 a-1} \Delta_{2 a+3}}{\left(\Delta_{2 a+1}\right)^{2}} \\
& \left\{\begin{array}{ccc}
2 a & 2 & 2 a \\
2 a \pm 2 & 2 & 2 a
\end{array}\right\}=\frac{d}{\Delta_{2 a \pm 1}} \quad\left\{\begin{array}{lll}
2 a & 2 & 2 a \pm 2 \\
2 a & 2 & 2 a \pm 2
\end{array}\right\}=\frac{d}{\Delta_{2 a \pm 1} \Delta_{2 a}} \\
& \left\{\begin{array}{ccc}
2 a & 2 & 2 a-2 \\
2 a & 2 & 2 a
\end{array}\right\}=\frac{\Delta_{2 a-2} \Delta_{2 a+1}}{\left(\Delta_{2 a-1}\right)^{2} \Delta_{2 a}} \quad\left\{\begin{array}{lll}
2 a & 2 & 2 a-2 \\
2 a & 2 & 2 a+2
\end{array}\right\}=\frac{\Delta_{2 a-2} \Delta_{2 a+2}}{\left(\Delta_{2 a}\right)^{2}}
\end{aligned}
$$

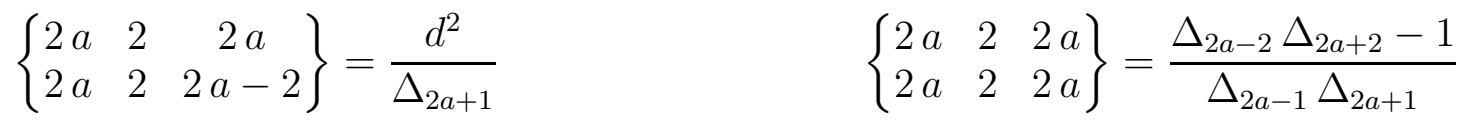

$$
\begin{aligned}
& \left\{\begin{array}{ccc}
2 a & 2 & 2 a \\
2 a & 2 & 2 a+2
\end{array}\right\}=-\frac{d^{2} \Delta_{2 a-1} \Delta_{2 a+2}}{\left(\Delta_{2 a+1}\right)^{2} \Delta_{2 a}} \quad\left\{\begin{array}{ccc}
2 a & 2 & 2 a+2 \\
2 a & 2 & 2 a
\end{array}\right\}=-\frac{1}{\Delta_{2 a-1}} \\
& \left\{\begin{array}{lll}
2 a & 2 & 2 a+2 \\
2 a & 2 & 2 a-2
\end{array}\right\}=\left\{\begin{array}{ccc}
2 a & 2 & 2 a \\
2 a-2 & 2 & 2 a-2
\end{array}\right\}=1 \\
& \left\{\begin{array}{ccc}
2 a & 2 & 2 a-2 \\
2 a-2 & 2 & 2 a-2
\end{array}\right\}=-\frac{1}{\Delta_{2 a-1}} \quad\left\{\begin{array}{ccc}
2 a & 2 & 2 a-2 \\
2 a-2 & 2 & 2 a
\end{array}\right\}=\frac{\Delta_{2 a-3} \Delta_{2 a+1}}{\left(\Delta_{2 a-1}\right)^{2}}
\end{aligned}
$$

It should be remarked that, in Ref. 11, studied was the quantum spin Hamiltonian acting on state (7.19) with spin-1 for the $S U(2)_{3}$ theory. 


\subsection{Topological Entanglement Entropy of Many-Quasi-Particle States}

We first discuss the entanglement entropy of the 4-quasi-particle states $\left|i_{a b ; c d}\right\rangle$ (7.14). We set a state by

$$
|\Psi\rangle=\sum_{i} p_{i}\left|i_{a b ; c d}\right\rangle
$$

where $p_{i} \in \mathbb{C}$ and

$$
\sum_{i}\left|p_{i}\right|^{2}=1
$$

Note that the sum of $i$ runs over such that triples $(i, a, b)$ and $(i, c, d)$ are admissible (2.24). The density matrix is written as

$$
\rho=|\Psi\rangle\langle\Psi|=\sum_{i, j} p_{i} p_{j}^{*} \sqrt{\frac{\Delta_{i}}{\theta(a, b, i) \theta(c, d, i)} \frac{\Delta_{j}}{\theta(a, b, j) \theta(c, d, j)}}
$$

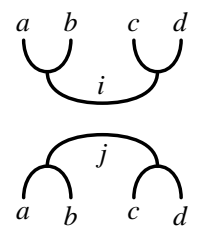

We first consider the entanglement between $A$ and $B$ when Alice owns spin- $a / 2$ and $-b / 2$ quasiparticles and Bob others; we write $A=\{a, b\}$ and $B=\{c, d\}$. Alice's reduced density matrix is depicted as

$$
\begin{aligned}
\rho_{\{a, b\}} & =\sum_{i, j} p_{i} p_{j}^{*} \sqrt{\frac{\Delta_{i}}{\theta(a, b, i) \theta(c, d, i)} \frac{\Delta_{j}}{\theta(a, b, j) \theta(c, d, j)}} \\
& =\sum_{i}\left|p_{i}\right|^{2} \frac{1}{\theta(a, b, i)} \overbrace{a}^{a}
\end{aligned}
$$

where we have used (7.8) in the second equality. Then the entanglement entropy (3.5) is computed from (3.13) as

$$
S_{\{a, b\}}=-\sum_{i}\left|p_{i}\right|^{2} \log \left(\frac{\left|p_{i}\right|^{2}}{\Delta_{i}}\right)
$$

where we have used (7.8) again. The identity (7.8) indicates that the expression (7.14) has orthonormal bases in Alice's and Bob's spaces. Then we obtain the topological entanglement entropy (3.9) as

$$
S_{\{a, b\}}^{\mathrm{topo}}=\sum_{i}\left|p_{i}\right|^{2} \log \Delta_{i}
$$

Indeed $\Delta_{i}$ denotes the quantum dimension $d_{i}$ of the quasi-particle with spin- $i / 2$ (2.30) who intertwines Alice $\{a, b\}$ and Bob $\{c, d\}$. 
To study a case when Alice has spin- $b / 2$ and $-c / 2$ quasi-particles, $A=\{b, c\}$ and $B=\{a, d\}$, we prepare different bases using the quantum $6 j$-symbol (7.1). We have

$$
|\Psi\rangle=\sum_{k} \widetilde{p_{k}}\left|\widetilde{k_{a b: c d}}\right\rangle
$$

where

$$
\begin{aligned}
& \left|\widetilde{k_{a b: c d}}\right\rangle=\sqrt{\frac{\Delta_{k}}{\theta(k, b, c) \theta(k, a, d)}} \bigcup_{k}^{a} j^{d} \\
& \widetilde{p_{k}}=\sum_{i} p_{i} \sqrt{\frac{\Delta_{i} \theta(b, c, k) \theta(a, d, k)}{\Delta_{k} \theta(a, b, i) \theta(c, d, i)}}\left\{\begin{array}{lll}
a & b & k \\
c & d & i
\end{array}\right\}
\end{aligned}
$$

Using $\left\{\begin{array}{lll}a & b & i \\ c & d & j\end{array}\right\}^{*}=\left\{\begin{array}{lll}b & a & i \\ d & c & j\end{array}\right\}$, the orthogonal identities (7.11), and symmetries (7.9) and (7.10), we indeed see that

$$
\sum_{k}\left|\widetilde{p_{k}}\right|^{2}=1
$$

Then one sees that Alice's reduced density matrix is computed as

$$
\begin{aligned}
\rho_{\{b, c\}} & =\sum_{j, k} \widetilde{p_{k}}{\widetilde{p_{j}}}^{*} \sqrt{\frac{\Delta_{k}}{\theta(b, c, k) \theta(a, d, k)} \frac{\Delta_{j}}{\theta(b, c, j) \theta(a, d, j)}} \\
& =\sum_{k}\left|\widetilde{p_{k}}\right|^{2} \frac{1}{\theta(b, c, k)} \overbrace{k}^{b} d \underbrace{c}_{c}
\end{aligned}
$$

which gives

$$
S_{\{b, c\}}=-\sum_{k}\left|\widetilde{p_{k}}\right|^{2} \log \left(\frac{\left|\widetilde{p_{k}}\right|^{2}}{\Delta_{k}}\right)
$$

By the same argument, we can identify the topological entanglement entropy with

$$
S_{\{b, c\}}^{\text {topo }}=\sum_{k}\left|\widetilde{p_{k}}\right|^{2} \log \Delta_{k}
$$

which shows that we have the quantum dimension of quasi-particle which intertwines Alice and Bob in the expression (7.36).

It is straightforward to study a case when Alice has only a spin- $a / 2$ quasi-particle; $A=\{a\}$ and $B=\{b, c, d\}$. From (7.32), we have Alice's reduced density matrix as

$$
\rho_{\{a\}}=\frac{1}{\Delta_{a}} \stackrel{a}{ }
$$

which gives

$$
S_{\{a\}}^{\text {topo }}=S_{\{a\}}=\log \Delta_{a}
$$


As the entanglement entropy (3.5) satisfies (3.4), we may expect

$$
S_{\{a, b, c\}}^{\text {topo }}=S_{\{a, b, c\}}=\log \Delta_{d}
$$

We shall check this formula skein-theoretically. The reduced density matrix is given by

$$
\rho_{\{a, b, c\}}=\sum_{i, j} m_{i, j} \overbrace{a}^{j} \overbrace{c}^{b}
$$

where

$$
m_{i, j}=p_{i} p_{j}^{*} \sqrt{\frac{\Delta_{i}}{\theta(a, b, i) \theta(c, d, i)} \frac{\Delta_{j}}{\theta(a, b, j) \theta(c, d, j)}}
$$

Then we have

$$
\begin{aligned}
\operatorname{Tr}_{\{a, b, c\}}\left(\rho_{\{a, b, c\}}\right)^{n} & =\sum_{j_{1}, \ldots, j_{n}} m_{j_{n}, j_{1}} m_{j_{1}, j_{2}} \cdots m_{j_{n-1}, j_{n}}\left(\prod_{k=1}^{n} \frac{\theta\left(j_{k}, a, b\right) \theta\left(j_{k}, c, d\right)}{\Delta_{j_{k}} \Delta_{d}}\right) \Delta_{d} \\
& =\sum_{j_{1}, \ldots, j_{n}}\left|p_{j_{1}}\right|^{2} \cdots\left|p_{j_{n}}\right|^{2} \frac{1}{\Delta_{d}^{n-1}} \\
& =\frac{1}{\Delta_{d}^{n-1}}
\end{aligned}
$$

The replica trick (3.8) gives (7.43) as we expected.

We stress again the the topological entanglement entropy $S_{A}^{\text {topo }}$ also depends on how to define $A$ and $B$.

To conclude we have shown that the topological entanglement entropy is written in terms of the quantum dimension. Namely in the case that the state $|\Psi\rangle$ with non-Abelian quasi-particles has a form (3.1), the entanglement entropy is modified to

$$
S_{A}=\sum_{j}\left|p_{j}\right|^{2} \log \left(\frac{d_{j}}{\left|p_{j}\right|^{2}}\right)
$$

which includes the topological entanglement entropy

$$
S_{A}^{\text {topo }}=\sum_{j}\left|p_{j}\right|^{2} \log d_{j}
$$

where $\left|p_{j}\right|^{2}$ denotes a probability, $\sum_{j}\left|p_{j}\right|^{2}=1$, that, in the state $|\Psi\rangle$, quasi-particle with quantum dimension $d_{j}$ plays a role of Cupid who intertwines Alice and Bob. We have obtained (7.47) for 4-quasi-particle states, but a computation for many-quasi-particle states is same because recursive use of the identity (7.7) enables us to rewrite any braided trivalent graphs into a graph where only a single Wilson line intertwines Alice and Bob. Once we have such trivalent graph, the identity (7.8) proves that we indeed have orthogonal bases in a sense of (3.2). So our formula (7.47) for the topological entanglement entropy is correct for other many-quasi-particle states. 


\section{Concluding Remarks and Discussions}

We have studied the topological properties of many-quasi-particle states in the Read-Rezayi state whose effective theory is the $S U(2)_{K}$ Chern-Simons theory. Applying the skein theory, we have obtained the unitary representation of the braiding matrices of quasi-particle states. These braiding matrices can be used as fundamental gates to construct other unitary gates. For example, by use of braiding matrices (4.31) of the 4-quasi-particle states in the Pfaffian state, we can construct the NOT gate and the Hadamard gate by [20]

$$
\begin{gathered}
\rho\left(\theta_{1}^{2} \sigma_{2}^{2}\right)=\left(\begin{array}{ll}
0 & 1 \\
1 & 0
\end{array}\right) \\
\rho\left(\theta_{1} \sigma_{1} \sigma_{2} \sigma_{1}\right)=\frac{-1}{\sqrt{2}}\left(\begin{array}{cc}
1 & 1 \\
1 & -1
\end{array}\right)
\end{gathered}
$$

Unfortunately as was shown in Refs. 13,14 this $S U(2)_{2}$ theory is not universally computable, although proposed in Ref. 5 to use another non-topological gate for universal computations. The simplest non-Abelian theory which is responsible for universal computation is the Fibonacci anyon model, i.e., $S U(2)_{3}$ theory. The Solovay-Kitaev algorithm [37, 47] supports that any unitary gates can be constructed from braiding matrices (5.25) and (5.26) of the Fibonacci anyons, although it was shown [16] that the gate of large Fourier transformation cannot be realized exactly. In fact several unitary gates were approximated using these braiding matrices [4, 27, 55]. It may be interesting to construct fundamental gates such as NOT, Hadamard, and CNOT, using the braiding matrices of $S U(2)_{K}$ theory presented here.

Using the braided trivalent graph as bases of Hilbert space of many-quasi-particle states, we have proposed a method to compute the bipartite entanglement entropy. Topological effect to the entanglement entropy was discussed in Refs. 35, 39, but extraction of topological part from the entanglement entropy is generally subtle in computations in physical models such as the quantum dimer model [18, 48]. Our construction is suitable to avoid such subtlety due to that the skein theory or the Chern-Simons theory is purely topological gauge theory. We have indeed obtained exactly topological contribution to the entanglement entropy. We have shown that manyquasi-particle states have the topological entanglement entropy (7.47) which depends only on the quantum dimension of a quasi-particle intertwining Alice and Bob.

\section{ACKNOWLEDGMENTS}

This work is supported in part by Grant-in-Aid from the Ministry of Education, Culture, Sports, Science and Technology of Japan.

\section{REFERENCES}

[1] L. Amico, R. Fazio, A. Osterloh, and V. Vedral, Entanglement in many-body systems, quantph/0703044 (2007). 
[2] E. Ardonne and K. Schoutens, Wavefunctions for topological quantum registers, Ann. Phys. 322, 201-235 (2007).

[3] P. Bonderson, K. Shtengel, and J. K. Slingerland, Interferometry of non-Abelian anyons, arXiv:0707.4206 (2007).

[4] N. E. Bonesteel, L. Hormozi, G. Zikos, and S. H. Simon, Braid topologies for quantum computation, Phys. Rev. Lett. 95, 140503 (2005).

[5] S. Bravyi, Universal quantum computation with the $\nu=5 / 2$ fractional quantum Hall state, Phys. Rev. A 73, 042313 (2006), 16 pages.

[6] P. Calabrese and J. Cardy, Entanglement entropy and quantum field theory, J. Stat. Mech. Theory Exp. 0406, P002 (2004), 27 pages.

[7] J. S. Carter, D. Flath, and M. Saito, Classical and Quantum $6 j$ Symbols, Princeton Univ. Press, Princeton, 1995.

[8] N. R. Cooper, N. K. Wilkin, and J. M. F. Gunn, Quantum phases of vortices in rotating Bose-Einstein condensates, Phys. Rev. Lett. 87, 120405 (2001).

[9] S. Das Sarma, M. Freedman, C. Nayak, S. H. Simon, and A. Stern, Non-Abelian anyons and topological quantum computation, arXiv:0707.1889 (2007).

[10] P. Di Francesco, P. Mathieu, and D. Sénéchal, Conformal Field Theory, Springer, New York, 1997.

[11] A. Feiguin, S. Trebst, A. W. W. Ludwig, M. Troyer, A. Kitaev, Z. Wang, and M. H. Freedman, Interacting anyons in topological quantum liquids: The golden chain, Phys. Rev. Lett. 98, 160409 (2007).

[12] E. Fradkin, C. Nayak, A. Tsvelik, and F. Wilczek, A Chern-Simons effective field theory for the Pfaffian quantum Hall state, Nucl. Phys. B 516, 704-718 (1998).

[13] M. H. Freedman, M. J. Larsen, and Z. Wang, A modular functor which is universal for quantum computation, Commun. Math. Phys. 227, 605-622 (2002).

[14] - The two-eigenvalue problem and density of Jones representation of braid groups, Commun. Math. Phys. 228, 177-199 (2002).

[15] M. H. Freedman, C. Nayak, K. Shtengel, K. Walker, and Z. Wang, A class of P, T-invariant topological phases of interacting electrons, Ann. Phys. 310, 428-492 (2004).

[16] M. H. Freedman and Z. Wang, Large Fourier transforms never exactly realized by braiding conformal blocks, Phys. Rev. A 75, 032322 (2006), 5 pages.

[17] P. Freyd, D. Yetter, J. Hoste, W. B. R. Lickorish, K. Millett, and A. Ocneanu, A new polynomial invariant of knots and links, Bull. Amer. Math. Soc. 12, 239-246 (1985).

[18] S. Furukawa and G. Misguich, Topological entanglement entropy in the quantum dimer model on the triangular lattice, Phys. Rev. B 75, 214407 (2007), 10 pages.

[19] L. S. Georgiev, Topologically protected gates for quantum computation with non-Abelian anyons in the Pfaffian quantum Hall state, Phys. Rev. B 74, 235112 (2006), 6 pages.

[20] - Towards a universal set of topologically protected gates for quantum computation with Pfaffian qubits, hep-th/0611340 (2006).

[21] D. Gepner and Z. Qiu, Modular invariant partition functions for parafermionic field theories, Nucl. Phys. B 285, 423-453 (1987). 
[22] D. Gepner and E. Witten, String theory on group manifolds, Nucl. Phys. B 278, 493-549 (1986).

[23] C. Gómez, M. Ruiz-Altaba, and G. Sierra, Quantum Groups in Two-dimensional Physics, Cambdrige Univ. Press, Cambdridge, 1996.

[24] M. Greiter, X. G. Wen, and F. Wilczek, Paired Hall states, Nucl. Phys. B 374, 567-614 (1992).

[25] M. Haque, O. Zozulya, and K. Schoutens, Entanglement entropy in fermionic Laughlin states, Phys. Rev. Lett. 98, 060401 (2007).

[26] C. Holzhey, F. Larsen, and F. Wilczek, Geometric and renormalized entropy in conformal field theory, Nucl. Phys. B 424, 443-467 (1994).

[27] L. Hormozi, G. Zikos, N. E. Bonesteel, and S. H. Simon, Topological quantum compiling, Phys. Rev. B 75, 165310 (2007), 19 pages.

[28] D. A. Ivanov, Non-Abelian statistics of half-quantum vortices in p-wave superconductors, Phys. Rev. Lett. 86, 268-271 (2001).

[29] V. F. R. Jones, A polynomial invariant for knots via von Neumann algebras, Bull. Amer. Math. Soc. 12, 103-111 (1985).

[30] C. Kassel, M. Rosso, and V. Turaev, Quantum Groups and Knot Invariants, no. 5 in Panoramas et Synthéses, Société Mathématique de France, Paris, 1997.

[31] L. H. Kauffman and S. L. Lins, Temperley-Lieb Recoupling Theory and Invariants of 3Manifolds, vol. 134 of Ann. Math. Stud., Princeton Univ. Press, Princeton, 1994.

[32] L. H. Kauffman and S. J. Lomonaco, Jr., q-deformed spin networks, kont polynomials and anyonic topological quantum computation, J. Knot Theory Ramif. 16, 267-332 (2007).

[33] A. N. Kirillov and N. Y. Reshetikhin, Representations of the algebra $U_{q}(S L(2))$, qorthogonal polynomials and invariants of links, in V. G. Kac, ed., Infinite Dimensional Lie Algebra and Groups, pp. 285-339, World Scientific, Singapore, 1989.

[34] A. Kitaev, Anyons in an exactly solved model and beyond, Ann. Phys. 321, 2-111 (2006).

[35] A. Kitaev and J. Preskill, Topological entanglement entropy, Phys. Rev. Lett. 96, 110404 (2006).

[36] A. Y. Kitaev, Fault-tolerant quantum computation by anyons, Ann. Phys. 303, 2-30 (2003).

[37] A. Y. Kitaev, A. H. Shen, and M. N. Vyalyi, Classical and Quantum Computation, vol. 47 of Grad. Stud. Math., Amer. Math. Soc., Providence, 2002.

[38] V. G. Knizhnik and A. B. Zamolodchikov, Current algebra and Wess-Zumino model in two dimensions, Nucl. Phys. B 247, 83-103 (1984).

[39] M. Levin and X.-G. Wen, Detecting topological order in a ground state wave function, Phys. Rev. Lett. 96, 110405 (2006).

[40] M. A. Levin and X.-G. Wen, String-net condensation: a physical mechanism for topological phases, Phys. Rev. B 71, 045110 (2005), 21 pages.

[41] W. B. R. Lickorish, The skein method for three-manifold invariants, J. Knot Theory and its Ramifications 2, 171-194 (1993).

[42] — - An Introduction to Knot Theory, vol. 175 of Graduate Texts in Mathematics, Springer, New York, 1997. 
[43] G. Masbaum and P. Vogel, 3-valent graphs and the Kauffman bracket, Pacific J. Math. 164, 361-381 (1994).

[44] G. Moore and N. Read, Nonabelions in the fractional quantum Hall effect, Nucl. Phys. B 360, 362-396 (1991).

[45] G. Moore and N. Seiberg, Classical and quantum conformal field theory, Commun. Math. Phys. 123, 177-254 (1989).

[46] C. Nayak and F. Wilczek, 2n-quasihole states realize $2^{n-1}$-dimensional spinor braiding statistics in paired quantum Hall states, Nucl. Phys. B 479, 529-553 (1996).

[47] M. A. Nielsen and I. L. Chuang, Quantum Computation and Quantum Information, Cambridge Univ. Press, Cambridge, 2000.

[48] S. Papanikolau, K. S. Raman, and E. Fradkin, Topological phases and topological entropy of two-dimensional systems with finite correlation length, arXiv:0709.0729 (2007).

[49] J. Preskill, Lecture notes of physics 219: Chapter 9. topological quantum computation (2004), http: / / www.theory . caltech.edu/ preskill/ph219/

[50] N. Read, Order parameter and Ginzburg-Landau theory for the fractional quantum Hall effect, Phys. Rev. Lett. 62, 86-89 (1989).

[51] - Paired fractional quantum Hall states and the $\nu=5 / 2$ puzzle, Physica B 298, 121-128 (2001).

[52] N. Read and D. Green, Paired states of fermions in two dimensions with breaking of parity and time-reversal symmetries, and the fractional quantum Hall effect, Phys. Rev. B 61, 10267-10297 (2000).

[53] N. Read and E. Rezayi, Beyond paired quantum Hall states: parafermions and incompressible states in the first excited Landau level, Phys. Rev. B 59, 8084-8092 (1999).

[54] N. Y. Reshetikhin and V. G. Turaev, Invariants of 3-manifolds via link polynomials and quantum groups, Invent. Math. 103, 547-597 (1991).

[55] S. H. Simon, N. E. Bonesteel, M. H. Freedman, N. Petrovic, and L. Hormozi, Topological quantum computing with only one mobile quasiparticles, Phys. Rev. Lett. 96, 070503 (2006).

[56] J. K. Slingerland and F. A. Bais, Quantum groups and non-Abelian braiding in quantum Hall systems, Nucl. Phys. B 612, 229-290 (2001).

[57] E. Verlinde, Fusion rules and modular transformation in 2D conformal field theory, Nucl. Phys. B 300, 360-376 (1988).

[58] X.-G. Wen and Q. Niu, Ground state degeneracy of the FQH states in presence of random potential and on high genus Riemann surfaces, Phys. Rev. B 40, 9377-9395 (1990).

[59] F. Wilczek, ed., Fractional Statistics and Anyon Superconductivity, World Scientific, Singapore, 1990.

[60] R. Willett, J. P. Eisenstein, H. L. Störmer, D. C. Tsui, A. C. Gossard, and J. H. English, Observation of an even-denominator quantum number in the fractional quantum Hall effect, Phys. Rev. Lett. 59, 1776-1779 (1987).

[61] E. Witten, Quantum field theory and the Jones polynomial, Commun. Math. Phys. 121, 351399 (1989). 
[62] J. S. Xia, W. Pan, C. L. Vicente, E. D. Adams, N. S. Sullivan, H. L. Stormer, D. C. Tsui, L. N. Pfeiffer, K. W. Baldwin, and K. W. West, Electron correlation in the second Landau level: a competition between many nearly degenerate quantum phases, Phys. Rev. Lett. 93, 176809 (2004).

[63] A. B. Zamolodchikov and V. A. Fateev, Nonlocal (parafermion) currents in two-dimensional conformal quantum field theory and self-dual critical points in $Z_{N}$-symmetric statistical systems, Sov. Phys. JETP 62, 215-225 (1985).

[64] S.-C. Zhang, T. H. Hansson, and S. Kivelson, Effective-field-theory model for the fractional quantum Hall effect, Phys. Rev. Lett. 62, 82-85 (1989).

Department of Physics, Graduate School of Science, University of Tokyo, Hongo 7-3-1, BUNKYO, TOKYO 113-0033, JAPAN.

$U R L:$ http://gogh.phys.s.u-tokyo.ac.jp/ hikami/

E-mail address: hikami@phys.s.u-tokyo.ac.jp 